\title{
Rational Tuning of Visual Cycle Modulator Pharmacodynamics
}

\author{
Philip D. Kiser, ${ }^{1}$ Jianye Zhang, ${ }^{1}$ Mohsen Badiee, Junzo Kinoshita, Neal S. Peachey, \\ Gregory P. Tochtrop, and Krzysztof Palczewski \\ Department of Pharmacology, School of Medicine (P.D.K., J.Z., K.P.), Department of Chemistry (M.B., G.P.T.), Case Western \\ Reserve University, Cleveland, Ohio; Research Service, Louis Stokes Cleveland Veterans Affairs Medical Center, Cleveland, Ohio \\ (P.D.K., N.S.P.); Cole Eye Institute, Cleveland Clinic, Cleveland, Ohio (J.K., N.S.P.); and Department of Ophthalmology, \\ Cleveland Clinic Lerner College of Medicine of Case Western Reserve University, Cleveland, Ohio (N.S.P.)
}

Received February 11, 2017; accepted May 1, 2017

\begin{abstract}
Modulators of the visual cycle have been developed for treatment of various retinal disorders. These agents were designed to inhibit retinoid isomerase [retinal pigment epithelium-specific $65 \mathrm{kDa}$ protein (RPE65)], the rate-limiting enzyme of the visual cycle, based on the idea that attenuation of visual pigment regeneration could reduce formation of toxic retinal conjugates. Of these agents, certain ones that contain primary amine groups can also reversibly form retinaldehyde Schiff base adducts, which contributes to their retinal protective activity. Direct inhibition of RPE65 as a therapeutic strategy is complicated by adverse effects resulting from slowed chromophore regeneration, whereas effective retinal sequestration can require high drug doses with potential off-target effects. We hypothesized that the RPE65-emixustat crystal structure could help guide the design of retinaldehydesequestering agents with varying degrees of RPE65 inhibitory
\end{abstract}

activity. We found that addition of an isopropyl group to the central phenyl ring of emixustat and related compounds resulted in agents effectively lacking in vitro retinoid isomerase inhibitory activity, whereas substitution of the terminal 6-membered ring with branched moieties capable of stronger RPE65 interaction potentiated inhibition. The isopropyl derivative series produced discernible visual cycle suppression in vivo, albeit much less potently than compounds with a high affinity for the RPE65 active site. These agents were distributed into the retina and formed Schiff base adducts with retinaldehyde. Except for one compound [3-amino-1-(3-isopropyl-5-((2,6,6-trimethylcyclohex-1-en-1yl)methoxy)phenyl)propan-1-ol (MB-007)], these agents conferred protection against retinal phototoxicity, suggesting that both direct RPE65 inhibition and retinal sequestration are mechanisms of potential therapeutic relevance.

\section{Introduction}

Visual function in vertebrates relies on a vitamin A-derived molecule called retinaldehyde, which is the chromophore of rod and cone visual pigments (Hubbard and Wald, 1952). The efficacy of retinaldehyde in triggering light perception depends on it existing in an 11-cis configuration coupled to rod and cone opsins through a protonated imine (Schiff base) bond. Absorption of light by 11-cis-retinylidene stimulates its isomerization to an all-trans configuration with resultant conformational changes in the opsin molecule, allowing it to trigger phototransduction (Palczewski, 2006; Hofmann et al., 2009).

This research was supported in part by grants from the National Institutes of Health [Grants EY009339, EY027283, and EY024864 to K.P.; Grant CA157735 to G.P.T.; and Core Grants P30EY011373 and P30EY025585]; the Department of Veterans Affairs [Grant IK2BX002683 to P.D.K.; Research Career Scientist to N.S.P.); Research to Prevent Blindness; the National Science Foundation [Grant MRI-1334048] for NMR instrumentation; and the Arnold and Mabel Beckman Foundation. This work is based in part upon research conducted at the APS Northeastern Collaborative Access Team beamlines supported by the National Institutes of Health [Grants GM103403 and RR029205] and the Department of Energy [Grant DE-AC02-06CH11357]. K.P. is the John H. Hord Professor of Pharmacology.

K.P. is an inventor of U.S. Patent No. 8722669 ("Compounds and Methods of Treating Ocular Disorders") and U.S. Patent No. 20080275134 ("Methods for Treatment of Retinal Degenerative Disease") issued to Case Western Reserve University (CWRU), whose values may be affected by this publication. CWRU may license this technology for commercial development.

${ }^{1}$ P.D.K., J.Z., and M.B. contributed equally to this work.

https://doi.org/10.1124/jpet.117.240721.
The photoactivated visual pigment eventually releases alltrans-retinaldehyde, which terminates signal transduction and leaves the opsin molecule insensitive to further light stimulation. Restoration of light sensitivity depends upon the conversion of all-trans-retinaldehyde back into 11-cis-retinaldehyde (Wald, 1968). The classic version of this process, known as the visual cycle, is carried out by enzymes and retinoid-binding proteins located within and between the retinal pigment epithelium (RPE) and photoreceptor outer segments (Fig. 1A) (Kiser et al., 2014).

Despite its essential function in initiating vision, retinaldehyde can cause retinal toxicity, a feature implicated in diseases including Stargardt macular dystrophy and age-related macular degeneration (AMD) (Chen et al., 2012; Maeda et al., 2012). This toxicity stems in large part from its aldehyde functionality, which is reactive toward certain cellular nucleophiles (Ben-Shabat et al., 2002). At concentrations easily achieved in vivo under strong lighting conditions, retinaldehyde induces apoptosis in cultured cell lines (Maeda et al., 2009a; Chen et al., 2012). Mice deficient in ATP-binding cassette transporter A4 (ABCA4) and retinol dehydrogenase 8 (RDH8), key proteins involved in retinaldehyde clearance, are also susceptible to light-induced retinal degeneration (Maeda et al., 2008). These mice accumulate autofluorescent material in their retinas, known as lipofuscin, that is partially composed of retinaldehyde-phosphatidylethanolamine adducts, such as $N$-retinylidene- $N$-retinylethanolamine (A2E) (Sparrow et al., 2003). 
A

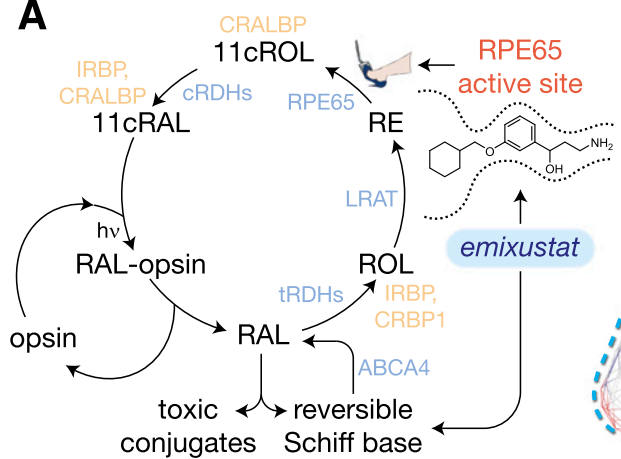

B

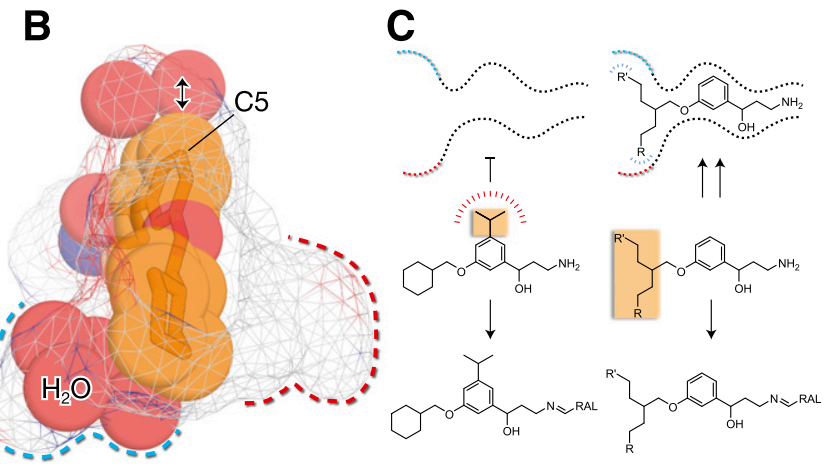

Fig. 1. Strategies for selective modulation of the retinoid cycle. (A) Emixustat modulates visual cycle function both by inhibition of 11-cis-retinol production through direct binding to the RPE65 active-site pocket, as well as by temporary sequestration of retinaldehyde through the formation of a Schiff base conjugate. Both modes of action can theoretically suppress the formation of toxic conjugates between retinaldehyde and cellular constituents that trigger retinal degeneration. Enzymes and retinoid-binding proteins of the visual cycle pathway are shown in blue- and peach-colored text, respectively. 11cRAL, 11-cis-retinal; 11cROL, 11-cis-retinol; CRALBP, cellular retinaldehyde-binding protein; CRBP1, cellular retinol-binding protein 1; tRDH, 11-cis-retinol dehydrogenase; IRBP, interphotoreceptor retinoid-binding protein; LRAT, lecithin:retinol acyltransferase; RAL, all-transretinaldehyde; RE, all-trans-retinyl ester; ROL, all-trans-retinol; RAL, all-trans-retinol dehydrogenase. (B) Structure of the emixustat-binding region of the RPE65 active-site cavity. Ligands and water molecules are shown as full-scale van der Waals spheres with carbon, nitrogen, and oxygen atoms colored orange, blue, and red, respectively, whereas the binding pocket is shown as a Connolly surface. The arrow emphasizes the relatively snug fit of the phenyl ring in the vicinity of carbon 5 (C5) to the active-site pocket. The water-filled polar (left) and apolar (right) binding pockets are delineated with blue and red dashed lines, respectively, on opposite sides of the emixustat cyclohexyl ring. This figure was generated using coordinates deposited under the Protein Data Bank accession code 4RSC in PyMOL (Schrödinger, LLC). (C) Structure-guided strategies to promote or block either of the two modes of visual cycle modulation exerted by emixustat. In the left panel, addition of a bulky hydrophobic substituent to the central phenyl ring should sterically prevent binding of the emixustat derivative to the RPE65 active-site pocket while simultaneously preserving RAL sequestration activity. In the right panel, design of inhibitors that occupy unused pockets within the RPE65 active-site cavity could improve potency and thus enable more selective RPE65 inhibition at lower drug concentrations.

Among strategies for preventing retinaldehyde toxicity, inhibition of the visual cycle was one of the first investigated and has been advanced to clinical testing (Fig. 1A). This approach is based on the observation that retinal pigment epithelium-specific $65 \mathrm{kDa}$ protein (RPE65)-null mice fail to develop retinal lipofuscin and A2E (Katz and Redmond, 2001). RPE65 catalyzes the rate-limiting trans-cis isomerization step of the visual cycle (Redmond et al., 1998, 2005). Inhibition of its activity was proposed to slow visual pigment regeneration, with less retinaldehyde being released during light exposure, thereby reducing lipofuscin formation (Maiti et al., 2006). This pharmacological concept was realized with the development of retinylamine, a transition state analog of the retinoid isomerization reaction (Golczak et al., 2005). A nonretinoid derivative of retinylamine, emixustat [ACU-4429 (3-amino-1(3-(cyclohexylmethoxy)phenyl)propan-1-ol)], was developed later (Bavik et al., 2015), entering clinical trials for the treatment of dry AMD (Kubota et al., 2012, 2014). It is currently being evaluated as a treatment of other conditions, including diabetic retinopathy (NCT02753400).
Both retinylamine (Maeda et al., 2011) and emixustat (Zhang et al., 2015) exert a second mechanism of protection against retinaldehyde toxicity whereby their primary amine groups reversibly react with retinaldehyde to form a Schiff base conjugate, thus quenching its reactivity (Fig. 1A). Manageable amounts of retinaldehyde are subsequently released over time to re-enter the visual cycle. This sequestration concept has been extended to include nonretinoid primary amine drugs that completely lack RPE65 inhibitory activity (Maeda et al., 2011).

Although compounds exhibiting either of these mechanisms of visual cycle modulation have the potential to ameliorate disease processes, their mechanisms of action make them inherently susceptible to producing untoward effects. Nyctalopia and dyschromatopsia, two adverse effects reported in emixustat clinical trials (Kubota et al., 2012, 2014), are direct extensions of visual cycle suppression. Long-term suppression of visual cycle activity is also linked to cone photoreceptor pathology in mice (Rohrer et al., 2005; Maeda et al., 2009b). On the other hand, the potentially high doses of primary amine

ABBREVIATIONS: ABCA4, ATP-binding cassette transporter A4; A2E, $N$-retinylidene- $N$-retinylethanolamine; AMD, age-related macular degeneration; compound 1, 2-propylpentyl methanesulfonate; compound 2, (R)-2-(3-hydroxy-3-(3-((2-propylpentyl)oxy)phenyl)propyl)isoindoline-1,3-dione; compound 4c, 3bromo-5-isopropylphenol; compound 5a, 1-bromo-3-(cyclohexylmethoxy)-5-isopropylbenzene; compound 5b, 1-bromo-3-isopropyl-5-((2-propylpentyl)oxy) benzene; compound 5c, 1-bromo-3-isopropyl-5-((2,6,6-trimethylcyclohex-1-en-1-yl)methoxy)benzene; compound 6a, 3-(cyclohexylmethoxy)-5isopropylbenzaldehyde; compound 6b, 3-isopropyl-5-((2-propylpentyl)oxy)benzaldehyde; compound 6c, 3-isopropyl-5-((2,6,6-trimethylcyclohex1-en-1-yl)methoxy)benzaldehyde; compound 7a, 3-(3-(cyclohexylmethoxy)-5-isopropylphenyl)-3-hydroxypropanenitrile; compound 7b, 3-hydroxy-3-(3-isopropyl-5-((2-propylpentyl)oxy)phenyl)propanenitrile; compound 7c, 3-hydroxy-3-(3-isopropyl-5-((2,6,6-trimethylcyclohex-1-en-1-yl)methoxy)phenyl)propanenitrile; compound 8, 3-hydroxy-3-(3-hydroxyphenyl)propanenitrile; compound 9, 1-bromopentan-2-one; compound 10, 3hydroxy-3-(3-((2-oxopentyl)oxy)phenyl)propanenitrile; compound 11, ethyl-3-((3-(2-cyano-1-hydroxyethyl)phenoxy)methyl)hex-2-enoate; compound 12, ethyl 3-((3-(2-cyano-1-hydroxyethyl)phenoxy)methyl)hexanoate; DMF, dimethylformamide; emixustat (ACU-4429), 3-amino-1-(3-(cyclohexylmethoxy) phenyl)propan-1-ol; eq., equivalent; ERG, electroretinogram; ESI-HRMS, Electrospray ionization-high resolution mass spectrometry; HPLC, high-performance liquid chromatography; MB-001, (R)-3-Amino-1-(3-((2,6,6-trimethylcyclohex-1-en-1-yl)methoxy)phenyl)propan-1-ol; MB-004, (R)-3-amino-1-(3-((2-propylpentyl)oxy)phenyl)propan-1-ol; MB-005, 3-amino-1-(3-(cyclohexylmethoxy)-5-isopropylphenyl)propan-1-ol; MB-006, 3amino-1-(3-isopropyl-5-((2-propylpentyl)oxy)phenyl)propan-1-ol; MB-007, 3-amino-1-(3-isopropyl-5-((2,6,6-trimethylcyclohex-1-en-1-yl)methoxy)phenyl) propan-1-ol; MB-008, 3-((3-(3-amino-1-hydroxypropyl)phenoxy)methyl)hexan-1-ol; nBuLi, n-butyl lithium; RDH8, retinol dehydrogenase 8; RPE, retinal pigment epithelium; RPE65, retinal pigment epithelium-specific $65 \mathrm{kDa}$ protein, retinoid isomerase; THF, tetrahydrofuran. 
compounds needed for effective retinaldehyde-sequestration activity have the potential to produce off-target effects (Maeda et al., 2011).

Crystal structures of RPE65 in complex with emixustat and a derivative called MB-001 [(R)-3-Amino-1-(3-((2,6,6trimethylcyclohex-1-en-1-yl)methoxy)phenyl)propan-1-ol] have revealed molecular interactions governing the binding affinity of these compounds (Kiser et al., 2015; Zhang et al., 2015). We hypothesized that these structures could inform strategies to modify the affinity of emixustat derivatives toward the RPE65 active site with maintenance of retinaldehyde-sequestering activity. To experimentally test this hypothesis, we synthesized emixustat derivatives with various degrees of predicted affinity for the RPE65 active site and characterized their effects on the visual cycle and ability to protect against retinal phototoxicity.

\section{Materials and Methods}

\section{Analysis of the RPE65 Emixustat-Binding Site}

The atomic coordinates for an RPE65-emixustat complex structure were downloaded from the Protein Data Bank (accession code 4RSC). The structure was visualized and analyzed using PyMOL (Schrodinger LLC, New York, NY) and COOT (Debreczeni and Emsley, 2012). Cavity volumes were estimated by manually filling the cavities with water molecules in PyMOL and calculating the volume of the resulting pseudomolecule using the Molinspiration Web server (http://www.molinspiration.com/services/volume.html). The same Web service was used to estimate the volumes of selected moieties within MB compounds.

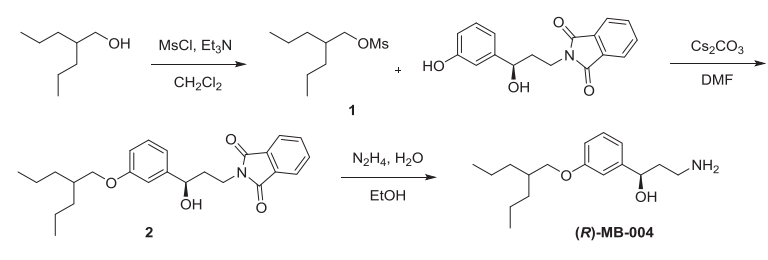

Scheme 1. Synthesis of (R)-MB-004.

\section{Synthetic Chemistry}

2-Propylpentyl Methanesulfonate (1). An oven-dried 100-ml, two-necked, round-bottomed flask equipped with a magnetic stir bar was sealed under argon with two rubber septa. The flask was charged with anhydrous $\mathrm{CH}_{2} \mathrm{Cl}_{2}$ (32 ml), 2-propylpentan-1-ol (2.5 g, $19.2 \mathrm{mmol}, 1.0$ eq.), and triethylamine $\left(2.9 \mathrm{~g}, 28.7 \mathrm{mmol}, 1.5\right.$ eq.). This solution was cooled to $0^{\circ} \mathrm{C}$, and then methanesulfonyl chloride ( $2.4 \mathrm{~g}, 21.1 \mathrm{mmol}, 1.01 \mathrm{eq}$.) was added dropwise. The reaction mixture was warmed to room temperature over a period of 2 hours. An aqueous solution of $1 \mathrm{~N} \mathrm{HCl}$ was added and the layers separated. The organic layer was dried over $\mathrm{Na}_{2} \mathrm{SO}_{4}$ and concentrated under reduced pressure. Purification by silica gel column chromatography (EtOAc/hexanes, 10:90) afforded $3.3 \mathrm{~g}(83 \%)$ of the desired compound 1 as a pale-yellow oil [ ${ }^{1} \mathrm{H} \mathrm{NMR}\left(500 \mathrm{MHz}, \mathrm{CDCl}_{3}\right) \delta 4.12(\mathrm{~d}, J=5.5 \mathrm{~Hz}, 2 \mathrm{H}), 3.00$ (s, 3H), $1.74(\mathrm{p}, J=5.5 \mathrm{~Hz}, 1 \mathrm{H}), 1.42-1.26(\mathrm{~m}, 8 \mathrm{H}), 0.99-0.84(\mathrm{~m}, 6 \mathrm{H}) ;{ }^{13} \mathrm{C}$ NMR $\left.\left(125 \mathrm{MHz}, \mathrm{CDCl}_{3}\right) \delta 72.65,37.45,37.32,33.06,19.87,14.40\right]$.

(R)-2-(3-Hydroxy-3-(3-((2-propylpentyl)oxy)phenyl)propyl)isoindoline-1,3-dione (2). A dried round-bottomed flask equipped with a stir bar was sealed under argon. It was charged with succinimideprotected phenol, prepared as described (Kiser et al., 2015) (4.3 g, $14.4 \mathrm{mmol}, 1.0$ eq.) in $85 \mathrm{ml}$ of anhydrous dimethylformamide (DMF). Anhydrous $\mathrm{Cs}_{2} \mathrm{CO}_{3}(6.4 \mathrm{~g}, 19.7 \mathrm{mmol}, 1.4 \mathrm{eq}$.) was added to a stirred solution of phenol. Compound 1 ( $3.4 \mathrm{~g}, 16.4 \mathrm{mmol}, 1.1 \mathrm{eq}$.) was added to the phenolate solution, and the reaction mixture was stirred at $60^{\circ} \mathrm{C}$ under argon for 24 hours. The reaction mixture was partitioned between EtOAc and $25 \% \mathrm{NH}_{4} \mathrm{Cl}$ (aqueous), and the aqueous layer was further extracted with EtOAc. The combined organic layers were washed with brine, dried over anhydrous $\mathrm{MgSO}_{4}$, and concentrated under reduced pressure. Purification by silica gel column chromatography (EtOAc/hexanes, 30:70) afforded $2.3 \mathrm{~g}$ (40\%) of the desired compound 2 as a colorless oil $\left[{ }^{1} \mathrm{H}\right.$ NMR $\left(500 \mathrm{MHz}, \mathrm{CDCl}_{3}\right) \delta 7.86-7.82(\mathrm{~m}, 2 \mathrm{H}), 7.74-7.70(\mathrm{~m}, 2 \mathrm{H})$, $7.18(\mathrm{t}, J=7.9 \mathrm{~Hz}, 1 \mathrm{H}), 6.91-6.89(\mathrm{~m}, 1 \mathrm{H}), 6.88(\mathrm{~d}, J=7.8 \mathrm{~Hz}, 1 \mathrm{H}), 6.73$ $(\mathrm{dd}, J=8.2,2.3 \mathrm{~Hz}, 1 \mathrm{H}), 4.66(\mathrm{dd}, J=8.1,5.2 \mathrm{~Hz}, 1 \mathrm{H}), 3.91(\mathrm{t}, J=6.5 \mathrm{~Hz}$, $2 \mathrm{H}), 3.80(\mathrm{~d}, J=5.6 \mathrm{~Hz}, 2 \mathrm{H}), 2.12-2.06(\mathrm{~m}, 2 \mathrm{H}), 1.81-1.74(\mathrm{~m}, 1 \mathrm{H})$, $1.45-1.30(\mathrm{~m}, 8 \mathrm{H}), 0.94-0.88(\mathrm{~m}, 6 \mathrm{H}) ;{ }^{13} \mathrm{C} \mathrm{NMR}\left(125 \mathrm{MHz}, \mathrm{CDCl}_{3}\right) \delta$ $168.94,159.70,145.29,134.17,132.18,129.55,123.46,117.77,113.76$, 111.77, 71.41, 70.91, 37.71, 37.69, 35.05, 33.87, 20.14, and 14.60].

(R)-3-amino-1-(3-((2-propylpentyl)oxy)phenyl)propan-1-ol [(R)-MB-004]. An oven-dried one-necked, round-bottomed flask equipped with a magnetic stir bar was charged with compound $2(2.3 \mathrm{~g}, 5.9 \mathrm{mmol}$, 1.0 eq.), $150 \mathrm{ml}$ of $\mathrm{EtOH}$, and $\mathrm{N}_{2} \mathrm{H}_{4} \cdot \mathrm{H}_{2} \mathrm{O}$ (1.6 ml, $\left.50.9 \mathrm{mmol}\right)$. The reaction mixture was stirred at room temperature for 24 hours, clarified by passage through filter paper, and the resulting filtrate was concentrated under reduced pressure. Purification by silica gel column chromatography $\left(\mathrm{NH}_{4} \mathrm{OH} / \mathrm{MeOH} / \mathrm{CH}_{2} \mathrm{Cl}_{2}, 4: 20: 80\right)$ afforded $1.18 \mathrm{~g}(75 \%)$ of the final product as a colorless oil $\left[{ }^{1} \mathrm{H} \mathrm{NMR}\left(500 \mathrm{MHz}, \mathrm{CD}_{3} \mathrm{OD}\right) \delta 7.24(\mathrm{t}, J=\right.$ $7.9 \mathrm{~Hz}, 1 \mathrm{H}), 6.94(\mathrm{~s}, 1 \mathrm{H}), 6.92(\mathrm{~d}, J=7.8 \mathrm{~Hz}, 1 \mathrm{H}), 6.81(\mathrm{~d}, J=8.3 \mathrm{~Hz}, 1 \mathrm{H})$, $4.76(\mathrm{t}, J=6.6 \mathrm{~Hz}, 1 \mathrm{H}), 3.86(\mathrm{~d}, J=5.4 \mathrm{~Hz}, 2 \mathrm{H}), 3.01-2.87(\mathrm{~m}, 2 \mathrm{H}), 1.96(\mathrm{q}$, $J=6.9 \mathrm{~Hz}, 2 \mathrm{H}), 1.80(\mathrm{p}, J=5.7 \mathrm{~Hz}, 1 \mathrm{H}), 1.49-1.34(\mathrm{~m}, 8 \mathrm{H}), 0.96-0.90(\mathrm{~m}$, $6 \mathrm{H}) ;{ }^{13} \mathrm{C}$ NMR $\left(125 \mathrm{MHz}, \mathrm{CD}_{3} \mathrm{OD}\right) \delta 160.99,147.46,130.50,118.86$, $114.39,112.95,73.26,71.76,39.11,39.05,38.85,34.93,21.06,14.78$; Electrospray ionization-high resolution mass spectrometry (ESI-HRMS): $m / z$ calculated for $\mathrm{C}_{17} \mathrm{H}_{30} \mathrm{NO}_{2}^{+}[\mathrm{M}+\mathrm{H}]^{+}: 280.22711$, found: 280.22708].

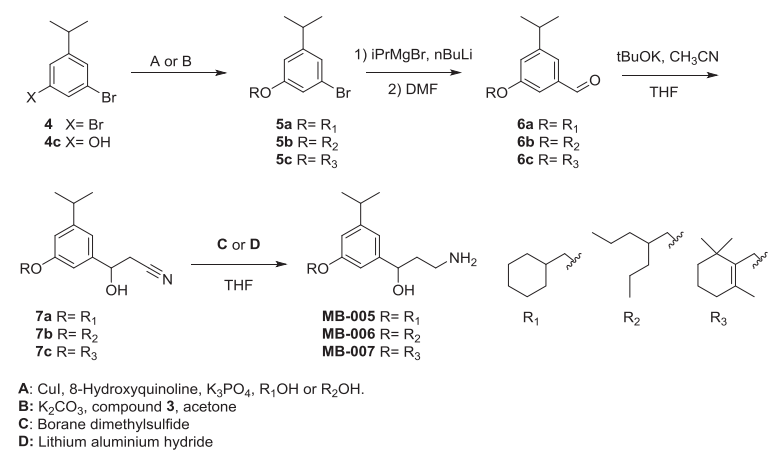

Scheme 2. Synthesis of MB-005, MB-006, and MB-007.

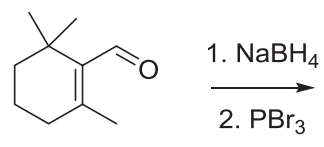<smiles>CC1=C(CBr)C(C)(C)CCC1</smiles>

3

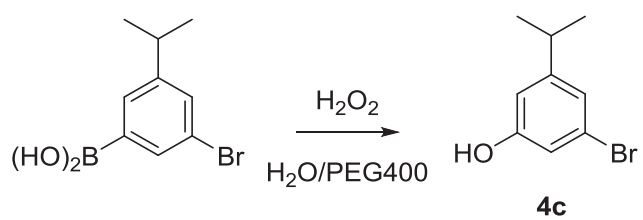

Scheme 3. Reagents for the synthesis of compounds 3 and 4c. Compound $\mathbf{3}$ was prepared as described (Crombie et al., 2001).

3-Bromo-5-isopropylphenol (4c). A 50-ml round-bottomed flask containing $16.48 \mathrm{ml}$ of CARBOWAX polyethylene glycol 400 (Dow, Midland, MI) was charged with $30 \%$ aqueous $\mathrm{H}_{2} \mathrm{O}_{2}(2.52 \mathrm{ml}$, $12.36 \mathrm{mmol}, 1.5$ eq.), and the resulting solution was stirred for 5 minutes at room temperature. Then, (3-bromo-5-isopropylphenyl) 
boronic acid ( $2 \mathrm{~g}, 8.24 \mathrm{mmol}, 1$ eq.) was added (note: reaction is highly exothermic) and stirred at room temperature open to air for a period of 2 hours. Upon completion of the reaction, the mixture was extracted by diethyl ether twice. The organic layers were combined, washed with brine, and dried over anhydrous $\mathrm{Na}_{2} \mathrm{SO}_{4}$, and the solvent was removed under reduced pressure (Gohain et al., 2014); purification by flash chromatography on a silica gel column (EtOAc/hexanes, 95:5) afforded $1.65 \mathrm{~g},(93 \%)$ of the desired phenol $4 \mathrm{c}$ as a white solid $\left[{ }^{1} \mathrm{H}\right.$ NMR $\left(500 \mathrm{MHz}, \mathrm{CDCl}_{3}\right) \delta 6.95(\mathrm{~s}, 1 \mathrm{H}), 6.83(\mathrm{~s}, 1 \mathrm{H}), 6.63(\mathrm{~s}, 1 \mathrm{H}), 4.79(\mathrm{~s}, 1 \mathrm{H})$, 2.82 (sept, $J=7.0 \mathrm{~Hz}, 1 \mathrm{H}), 1.21(\mathrm{~d}, J=6.9 \mathrm{~Hz}, 6 \mathrm{H}) ;{ }^{13} \mathrm{C} \mathrm{NMR}$ $\left(125 \mathrm{MHz}, \mathrm{CDCl}_{3}\right) \delta 156.30,152.72,122.68,122.48,116.25,112.62$, 34.12, 23.83] (Somai Magar and Lee, 2014).

1-Bromo-3-(cyclohexylmethoxy)-5-isopropylbenzene (5a). An ovendried 50-ml, pear-shaped Schlenk flask equipped with a magnetic stir bar was sealed under argon with a rubber septum (three cycles of evacuation followed by argon backfilling). The flask was charged with copper iodide $(514.2 \mathrm{mg}$, $2.7 \mathrm{mmol}, 0.15$ eq.), 8-hydroxyquinoline (784.2 $\mathrm{mg}, 5.4 \mathrm{mmol}, 0.3 \mathrm{eq}$. ), and anhydrous potassium phosphate tribasic $(7.65 \mathrm{~g}, 36.0 \mathrm{mmol}, 2.0 \mathrm{eq}$.). The flask was then evacuated and backfilled with argon (three cycles). Commercially available 1,3-dibromo-5-isopropylbenzene 4 (5 g, $18.0 \mathrm{mmol}, 1.0$ eq.) and cyclohexylmethanol $\left(\mathrm{R}_{1} \mathrm{OH}: 15 \mathrm{ml}\right)$ were then added at room temperature via a syringe. The rubber septum stopper was replaced with a screw cap, and the reaction mixture was placed in a preheated oil bath and stirred at $110^{\circ} \mathrm{C}$. After 24 hours, the reaction mixture was allowed to cool to $40^{\circ} \mathrm{C}$ and was then diluted with hexanes $(30 \mathrm{ml})$. After stirring 5 minutes at room temperature, the slurry was passed through filter paper, and the filter cake was washed with hexanes. The filtrate was concentrated under reduced pressure (Niu et al., 2009). Purification by flash column chromatography on a silica gel with $100 \%$ hexanes afforded $2.2 \mathrm{~g}$ (40\%) of the desired ether $5 \mathbf{a}$ as a colorless oil [ ${ }^{1} \mathrm{H} \mathrm{NMR}(500 \mathrm{MHz}$, $\left.\mathrm{CDCl}_{3}\right) \delta 6.94(\mathrm{~s}, 1 \mathrm{H}), 6.85(\mathrm{~s}, 1 \mathrm{H}), 6.69(\mathrm{~s}, 1 \mathrm{H}), 3.71(\mathrm{~d}, J=6.2 \mathrm{~Hz}, 2 \mathrm{H}), 2.81$ (sept, $J=7.1 \mathrm{~Hz}, 1 \mathrm{H}), 1.89-1.82(\mathrm{~m}, 2 \mathrm{H}), 1.82-1.67(\mathrm{~m}, 4 \mathrm{H}), 1.37-1.15(\mathrm{~m}, 3 \mathrm{H})$, $1.22(\mathrm{~d}, J=6.8 \mathrm{~Hz}, 6 \mathrm{H}), 1.09-1.00(\mathrm{~m}, 2 \mathrm{H}) ;{ }^{13} \mathrm{C} \mathrm{NMR}\left(125 \mathrm{MHz}, \mathrm{CDCl}_{3}\right) \delta$ $160.22,152.24,122.68,121.97,114.73,112.40,73.79,37.83,34.28,30.02,26.64$, 25.93, 23.90].

1-Bromo-3-isopropyl-5-((2-propylpentyl)oxy)benzene (5b). The same procedure was followed as described for 5a. In brief, the argon-filled flask was charged with copper iodide $(514.2 \mathrm{mg})$, 8-hydroxyquinoline (784.2 $\mathrm{mg}$ ), and anhydrous potassium phosphate tribasic $(7.65 \mathrm{~g})$. The flask was then evacuated and backfilled with argon (three cycles). Commercially available 1,3-dibromo-5isopropylbenzene 4 (5 g) and 2-propylpentan-1-ol $\left(\mathrm{R}_{2} \mathrm{OH}: 15 \mathrm{ml}\right)$ were added. Purification by flash column chromatography on silica gel with $100 \%$ hexanes afforded $1.95 \mathrm{~g}(33 \%)$ of the desired ether $\mathbf{5 b}$ as a colorless oil $\left[{ }^{1} \mathrm{H}\right.$ NMR $\left(500 \mathrm{MHz}, \mathrm{CDCl}_{3}\right) \delta 6.94(\mathrm{~s}, 1 \mathrm{H}), 6.86(\mathrm{~s}, 1 \mathrm{H})$, $6.69(\mathrm{~s}, 1 \mathrm{H}), 3.79(\mathrm{~d}, J=5.6 \mathrm{~Hz}, 2 \mathrm{H}), 2.83$ (sept, $J=7.2 \mathrm{~Hz}, 1 \mathrm{H}), 1.79$ (quint, $J=5.6 \mathrm{~Hz}, 1 \mathrm{H}), 1.47-1.31(\mathrm{~m}, 8 \mathrm{H}), 1.23(\mathrm{~d}, J=6.9 \mathrm{~Hz}, 6 \mathrm{H}), 0.92$ $(\mathrm{t}, J=6.9 \mathrm{~Hz}, 6 \mathrm{H}) ;{ }^{13} \mathrm{C} \mathrm{NMR}\left(125 \mathrm{MHz}, \mathrm{CDCl}_{3}\right) \delta 160.27,152.21$, $122.67,121.92,114.80,112.35,71.16,37.67,34.30,33.82,23.91,20.14$, $14.58]$.

1-Bromo-3-isopropyl-5-((2,6,6-trimethylcyclohex-1-en-1yl)methoxy)benzene (5c). An oven-dried 100-ml, three-necked, round-bottomed flask equipped with a magnetic stir bar was sealed under argon. The flask was charged with phenol 4c (1.83 g, $8.5 \mathrm{mmol}$, 1 eq.) and anhydrous potassium carbonate $(4.71 \mathrm{~g}, 34 \mathrm{mmol}, 4 \mathrm{eq}$.). The flask was evacuated, filled up with argon twice, and then charged with dry acetone $(60 \mathrm{ml})$. Compound $3(1.85 \mathrm{~g}, 8.5 \mathrm{mmol}, 1.2 \mathrm{eq}$.) was then added to this mixture. After reflux under argon atmosphere for 24 hours, the reaction mixture was cooled to room temperature and then filtered and concentrated under reduced pressure. Purification by flash chromatography on a silica gel column (100\% hexanes) afforded $2.4 \mathrm{~g}(80 \%)$ of the desired ether $\mathbf{5 c}$ as a colorless oil $\left[{ }^{1} \mathrm{H}\right.$ $\mathrm{NMR}\left(500 \mathrm{MHz}, \mathrm{CDCl}_{3}\right) \delta 6.96(\mathrm{~s}, 1 \mathrm{H}), 6.93(\mathrm{t}, J=2.0 \mathrm{~Hz}, 1 \mathrm{H}), 6.75(\mathrm{t}$, $J=1.9 \mathrm{~Hz}, 1 \mathrm{H}), 4.39(\mathrm{~s}, 2 \mathrm{H}), 2.83(\mathrm{sept}, J=6.9 \mathrm{~Hz}, 1 \mathrm{H}), 2.05(\mathrm{t}, J=$ $6.3 \mathrm{~Hz}, 2 \mathrm{H}), 1.70(\mathrm{~s}, 3 \mathrm{H}), 1.68-1.62(\mathrm{~m}, 2 \mathrm{H}), 1.53-1.48(\mathrm{~m}, 2 \mathrm{H}), 1.23(\mathrm{~d}$, $J=6.9 \mathrm{~Hz}, 6 \mathrm{H}), 1.04(\mathrm{~s}, 6 \mathrm{H}) ;{ }^{13} \mathrm{C} \mathrm{NMR}\left(125 \mathrm{MHz} \mathrm{CDCl}_{3}\right) \delta 160.12$, 152.08, 136.07, 132.83, 122.50, 121.92, 114.63, 112.61, 64.66, 39.24, $34.15,34.10,32.88,28.43,23.77,19.82,19.27]$.
3-(Cyclohexylmethoxy)-5-isopropylbenzaldehyde (6a). An oven-dried 50-ml, two-necked, round-bottomed flask was equipped with a magnetic stir bar and sealed under argon. The flask was charged with the aryl bromide $\mathbf{5 a}(2.5 \mathrm{~g}, 8.0 \mathrm{mmol}, 1.2 \mathrm{eq}$.) in anhydrous tetrahydrofuran (THF) $(8 \mathrm{ml})$. The reaction mixture was cooled to $0^{\circ} \mathrm{C}$, and a $3 \mathrm{M}$ solution of isopropylmagnesium bromide in 2-Met-THF ( $3.3 \mathrm{mmol}, 0.5$ eq.) was added over 5 minutes and stirred for an additional 10 minutes. Then a $2.16 \mathrm{M}$ solution of n-butyl lithium ( $\mathrm{nBuLi})$ in hexanes $(6.7 \mathrm{mmol}$, 1.0 eq.) was added dropwise over 10 minutes while the temperature was maintained below $5^{\circ} \mathrm{C}$. The resulting mixture was stirred at $0^{\circ} \mathrm{C}$ for 1 hour, cooled to $-10^{\circ} \mathrm{C}$, and dry DMF $(670 \mu \mathrm{l}, 8.9 \mathrm{mmol}, 1.3$ eq. $)$ in dry THF $(9 \mathrm{ml})$ was added dropwise over 10 minutes. The resulting mixture was warmed to room temperature over 1 hour, then added to a $0.5 \mathrm{M}$ citric acid solution $(30 \mathrm{ml})$. After 10-minute stirring, the phases were separated, and the aqueous phase was extracted one additional time with toluene. The combined organic phases were concentrated, and water was removed azeotropically with toluene (Gallou et al., 2008). Purification by flash chromatography on a silica gel column ( $\mathrm{Et}_{2} \mathrm{O} /$ hexanes, $\left.7: 93\right)$ afforded $1.9 \mathrm{~g}$ (90\%) of the desired aldehyde $6 \mathbf{a}$ as a colorless oil $\left[{ }^{1} \mathrm{H}\right.$ NMR $\left(500 \mathrm{MHz}, \mathrm{CDCl}_{3}\right) \delta 9.94(\mathrm{~s}, 1 \mathrm{H}), 7.32(\mathrm{~s}, 1 \mathrm{H}), 7.19(\mathrm{~s}, 1 \mathrm{H})$, $7.05(\mathrm{~s}, 1 \mathrm{H}), 3.80(\mathrm{~d}, J=6.3,2 \mathrm{H}), 2.95$ (sept, $J=7.2,6.8 \mathrm{~Hz}, 1 \mathrm{H})$, $1.92-1.85(\mathrm{~m}, 2 \mathrm{H}), 1.84-1.67(\mathrm{~m}, 4 \mathrm{H}), 1.36-1.17(\mathrm{~m}, 3 \mathrm{H}), 1.28(\mathrm{~d}, J=$ $6.9 \mathrm{~Hz}, 6 \mathrm{H}), 1.12-1.02(\mathrm{~m}, 2 \mathrm{H}) ;{ }^{13} \mathrm{C}$ NMR $\left(125 \mathrm{MHz}^{-} \mathrm{CDCl}_{3}\right) \delta 192.72$, $160.10,151.52,137.92,121.84,120.66,110.20,73.87,37.83,34.12$, $30.03,26.63,25.93,23.92]$.

3-Isopropyl-5-((2-propylpentyl)oxy)benzaldehyde $(6 \mathrm{~b})$. The same procedure was followed as described for $6 \mathbf{a}$. At $0^{\circ} \mathrm{C}$, a $3 \mathrm{M}$ solution of isopropylmagnesium bromide in 2-Met-THF ( $2.9 \mathrm{mmol}, 0.5$ eq.) was added to a solution of the aryl bromide $5 \mathbf{b}(2.27 \mathrm{~g}, 6.94 \mathrm{mmol}$, 1.2 eq.) in anhydrous THF (6.94 ml). Then, a $2.16 \mathrm{M}$ solution of $\mathrm{nBuLi}$ in hexanes ( $5.9 \mathrm{mmol}, 1.0 \mathrm{eq}$.) was added, followed by the addition of a solution of dry DMF (584 $\mu \mathrm{l}, 7.74 \mathrm{mmol}, 1.3 \mathrm{eq}$.$) in dry THF (7.74 \mathrm{ml})$ at $-10^{\circ} \mathrm{C}$. Purification by flash chromatography on a silica gel column $\left(\mathrm{Et}_{2} \mathrm{O} /\right.$ hexanes, $\left.7: 93\right)$ afforded $1.7 \mathrm{~g}(90 \%)$ of the desired aldehyde $\mathbf{6 b}$ as a colorless oil $\left[{ }^{1} \mathrm{H} \mathrm{NMR}\left(500 \mathrm{MHz}, \mathrm{CDCl}_{3}\right) \delta 9.95(\mathrm{~s}, 1 \mathrm{H}), 7.32(\mathrm{~s}, 1 \mathrm{H})\right.$, $7.21(\mathrm{~s}, 1 \mathrm{H}), 7.05(\mathrm{~s}, 1 \mathrm{H}), 3.88(\mathrm{~d}, J=5.3 \mathrm{~Hz}, 2 \mathrm{H}), 2.95$ (sept, $J=7.1 \mathrm{~Hz}$, $1 \mathrm{H}), 1.82(\mathrm{p}, J=5.8 \mathrm{~Hz}, 1 \mathrm{H}), 1.50-1.32(\mathrm{~m}, 8 \mathrm{H}), 1.28(\mathrm{~d}, J=7.0 \mathrm{~Hz}$, $6 \mathrm{H}), 0.95-0.89(\mathrm{~m}, 6 \mathrm{H}) ;{ }^{13} \mathrm{C} \mathrm{NMR}\left(125 \mathrm{MHz} \mathrm{CDCl}_{3}\right) \delta 192.64,160.14$, $151.47,137.92$, 121.74, 120.58, 110.27, 71.25, 37.66, 34.13, 33.82, $23.91,20.13,14.56]$.

3-Isopropyl-5-((2,6,6-trimethylcyclohex-1-en-1-yl)methoxy)benzaldehyde $(\mathbf{6 c})$. The same procedure was followed as described for $6 \mathbf{a}$. At $0^{\circ} \mathrm{C}$, a $3 \mathrm{M}$ solution of isopropylmagnesium bromide in 2-MetTHF (1.75 mmol, 0.5 eq.) was added to a solution of the aryl bromide $\mathbf{5 c}$ (1.47 g, $4.2 \mathrm{mmol}, 1.2$ eq.) in anhydrous THF ( $5 \mathrm{ml})$. Then, a $2.16 \mathrm{M}$ solution of $\mathrm{nBuLi}$ in hexanes (3.5 mmol, $1.0 \mathrm{eq}$.) was added, followed by the addition of a solution of dry DMF ( $343 \mu \mathrm{l}, 4.54 \mathrm{mmol}, 1.3 \mathrm{eq}$.) in dry $\mathrm{THF}(4.5 \mathrm{ml})$ at $-10^{\circ} \mathrm{C}$. Purification by flash chromatography on a silica gel column $\left(\mathrm{Et}_{2} \mathrm{O} /\right.$ hexanes, 5:95) afforded $1.1 \mathrm{~g}(87 \%)$ of the desired aldehyde $6 \mathbf{c}$ as a colorless oil $\left[{ }^{1} \mathrm{H} \mathrm{NMR}\left(500 \mathrm{MHz}, \mathrm{CDCl}_{3}\right) \delta 9.96(\mathrm{~s}, 1 \mathrm{H})\right.$, $7.34(\mathrm{t}, J=1.5 \mathrm{~Hz}, 1 \mathrm{H}), 7.29(\mathrm{dd}, J=2.6,1.3 \mathrm{~Hz}, 1 \mathrm{H}), 7.11(\mathrm{t}, J=2.1 \mathrm{~Hz}$, $1 \mathrm{H}), 4.48$ (s, 2H), 2.96 (sept, $J=6.9 \mathrm{~Hz}, 1 \mathrm{H}$ ), 2.06 (t, $J=6.3 \mathrm{~Hz}, 2 \mathrm{H}$ ), 1.72 $(\mathrm{s}, 3 \mathrm{H}), 1.69-1.63(\mathrm{~m}, 2 \mathrm{H}), 1.54-1.49(\mathrm{~m}, 2 \mathrm{H}), 1.28(\mathrm{~d}, J=6.9 \mathrm{~Hz}, 6 \mathrm{H})$, $1.06(\mathrm{~s}, 6 \mathrm{H}) ;{ }^{13} \mathrm{C} \mathrm{NMR}\left(125 \mathrm{MHz}, \mathrm{CDCl}_{3}\right) \delta 192.74,160.13,151.50$, 137.91 , 136.29, 132.99, 122.11, 121.06, 110.04, 64.89, 39.39, 34.27, $34.13,33.03,28.58,23.93,19.98,19.41]$.

3-(3-(Cyclohexylmethoxy)-5-isopropylphenyl)-3-hydroxypropanenitrile (7a). An oven-dried 50-ml, two-necked, round-bottomed flask was equipped with a magnetic stir bar and sealed under argon. The flask was charged with anhydrous THF $(9 \mathrm{ml})$ and anhydrous acetonitrile $\left(290 \mu \mathrm{l}, 5.2 \mathrm{mmol}, 1.01 \mathrm{eq}\right.$.), cooled to $-42^{\circ} \mathrm{C}$, and treated dropwise with a solution of potassium tert-butoxide $(630 \mathrm{mg}, 5.7 \mathrm{mmol}$, 1.12 eq.) in THF ( $6 \mathrm{ml})$. After stirring for 45 minutes, benzaldehyde $\mathbf{6 a}$ (1.3 g, $5 \mathrm{mmol}, 1.0$ eq.) in THF $(2.7 \mathrm{ml})$ was also added dropwise. The reaction was allowed to warm to $-15^{\circ} \mathrm{C}$ over a 4 -hour period and was quenched by the slow addition of $25 \% \mathrm{NH}_{4} \mathrm{Cl}$ (aqueous) $(1 \mathrm{ml}$ ). The layers were partitioned, and the aqueous phase was extracted twice with EtOAc. The combined organic layers were washed twice with $\mathrm{H}_{2} \mathrm{O}$ 
and once with brine, dried over $\mathrm{MgSO}_{4}$, and concentrated under reduced pressure. Purification by flash column chromatography on silica gel (EtOAc/hexanes, 35:65) afforded $1.3 \mathrm{~g}(86 \%)$ of the nitrile $\mathbf{7 a}$ as a pale-yellow oil $\left[{ }^{1} \mathrm{H}\right.$ NMR $\left(500 \mathrm{MHz}, \mathrm{CDCl}_{3}\right) \delta 6.80(\mathrm{~s}, 1 \mathrm{H}), 6.75(\mathrm{~s}$, $2 \mathrm{H}$ ), 4.99 (t, $J=6.3 \mathrm{~Hz}, 1 \mathrm{H}), 3.75(\mathrm{~d}, J=6.1 \mathrm{~Hz}, 2 \mathrm{H}), 2.87$ (sept, $J=$ $6.6 \mathrm{~Hz}, 1 \mathrm{H}), 2.78-2.74(\mathrm{~m}, 2 \mathrm{H}), 1.90-1.83(\mathrm{~m}, 2 \mathrm{H}), 1.81-1.66(\mathrm{~m}, 4 \mathrm{H})$, $1.36-1.25(\mathrm{~m}, 3 \mathrm{H}), 1.23(\mathrm{~d}, J=6.7 \mathrm{~Hz}, 6 \mathrm{H}), 1.11-1.01(\mathrm{~m}, 2 \mathrm{H}) ;{ }^{13} \mathrm{C} \mathrm{NMR}$ $\left(125 \mathrm{MHz}, \mathrm{CDCl}_{3}\right) \delta 160.03,151.56,142.52,117.48,115.89,113.49$, 108.61, 73.67, 70.59, 37.93, 34.41, 30.08, 28.04, 26.65, 25.95, 24.06, 24.01].

3-Hydroxy-3-(3-isopropyl-5-((2-propylpentyl)oxy)phenyl)propanenitrile (7b). The same procedure was followed as described for 7a. A solution of potassium tert-butoxide (770 mg, $6.9 \mathrm{mmol}, 1.12 \mathrm{eq}$.) in THF (7.5 ml) was added to a solution of anhydrous acetonitrile (356 $\mu \mathrm{l}, 6.4 \mathrm{mmol}, 1.04 \mathrm{eq}$.) in anhydrous $\mathrm{THF}(9 \mathrm{ml})$ at $-42^{\circ} \mathrm{C}$. Then, benzaldehyde $6 \mathrm{~b}$ (1.7 g, $6.2 \mathrm{mmol}, 1.0$ eq.) in THF (6 ml) was added dropwise, yielding the nitrile $\mathbf{7 b}(1.7 \mathrm{~g}, 87 \%)$ as a pale-yellow oil $\left[{ }^{1} \mathrm{H}\right.$ $\mathrm{NMR}\left(500 \mathrm{MHz} \mathrm{CDCl}_{3}\right) \delta 6.80(\mathrm{~s}, 1 \mathrm{H}), 6.75(\mathrm{~s}, 2 \mathrm{H}), 4.99(\mathrm{t}, J=6.4 \mathrm{~Hz}$, $1 \mathrm{H}), 3.82(\mathrm{~d}, J=5.5 \mathrm{~Hz}, 2 \mathrm{H}), 2.88$ (sept, $J=7.3 \mathrm{~Hz}, 1 \mathrm{H}), 2.79-2.75(\mathrm{~m}$, $2 \mathrm{H}), 2.30(\mathrm{bs}, 1 \mathrm{H}), 1.86-1.75(\mathrm{~m}, 1 \mathrm{H}), 1.48-1.32(\mathrm{~m}, 8 \mathrm{H}), 1.24(\mathrm{~d}, J=$ $6.7 \mathrm{~Hz}, 6 \mathrm{H}), 0.92(\mathrm{t}, J=6.8 \mathrm{~Hz}, 6 \mathrm{H}) ;{ }^{13} \mathrm{C} \mathrm{NMR}\left(125 \mathrm{MHz} \mathrm{CDCl}_{3}\right) \delta$ 159.95, 151.39, 142.37, 117.35, 115.71, 113.30, 108.55, 70.89, 70.47, $37.60,34.29,33.72,27.90,23.93,23.88,20.03,14.45]$.

3-Hydroxy-3-(3-isopropyl-5-((2,6,6-trimethylcyclohex-1-en-1yl)methoxy)phenyl)propanenitrile (7c). The same procedure was followed as described for 7a. A solution of potassium tert-butoxide (485 mg, $4.32 \mathrm{mmol}, 1.12 \mathrm{eq}$.) in THF (10 ml) was added to a solution of anhydrous acetonitrile ( $222 \mu \mathrm{l}, 4.2 \mathrm{mmol}, 1.1 \mathrm{eq}$.) in anhydrous THF $(8.4 \mathrm{ml})$ at $-42^{\circ} \mathrm{C}$. Then, benzaldehyde $6 \mathrm{c}$ ( $1.16 \mathrm{~g}, 3.9 \mathrm{mmol}, 1.0$ eq.) in THF ( $4 \mathrm{ml}$ ) was added dropwise. Purification by flash column chromatography on silica gel (EtOAc/hexanes, 35:65) afforded $0.9 \mathrm{~g}$ $(69 \%)$ of the desired nitrile $7 \mathbf{c}$ as a pale-yellow oil [ ${ }^{1} \mathrm{H} \mathrm{NMR}(500 \mathrm{MHz}$, $\left.\mathrm{CDCl}_{3}\right) \delta 6.84-6.82(\mathrm{~m}, 2 \mathrm{H}), 6.82-6.80(\mathrm{~m}, 1 \mathrm{H}), 5.01(\mathrm{t}, J=6.3 \mathrm{~Hz}, 1 \mathrm{H})$, $4.43(\mathrm{~s}, 2 \mathrm{H}), 2.89$ (sept, $J=6.8 \mathrm{~Hz}, 1 \mathrm{H}), 2.80-2.76(\mathrm{~m}, 2 \mathrm{H}), 2.29(\mathrm{~s}, 1 \mathrm{H})$, $2.05(\mathrm{~d}, J=6.3 \mathrm{~Hz}, 2 \mathrm{H}), 1.71(\mathrm{~s}, 3 \mathrm{H}), 1.69-1.62(\mathrm{~m}, 2 \mathrm{H}), 1.54-1.48(\mathrm{~m}$, $2 \mathrm{H}), 1.25(\mathrm{~d}, J=6.9 \mathrm{~Hz}, 6 \mathrm{H}), 1.05(\mathrm{~s}, 6 \mathrm{H}) ;{ }^{13} \mathrm{C} \mathrm{NMR}\left(125 \mathrm{MHz} \mathrm{CDCl}_{3}\right) \delta$ $160.08,151.53,142.47,136.06,133.13,117.51,116.00,113.81,108.75$, 70.66, 64.64, 39.40, 34.44, 34.26, 33.02, 28.60, 28.04, 24.08, 24.03, $19.99,19.42]$.

3-Amino-1-(3-(cyclohexylmethoxy)-5-isopropylphenyl)propan1-ol (MB-005). An oven-dried 50-ml, two-necked, round-bottomed flask equipped with a magnetic stir bar was sealed under argon. It had two rubber septa, one of which contained a needle adapter to an argon inlet. The flask was charged with anhydrous THF (14 ml) and nitrile $7 \mathrm{a}$ (1.3 g, $4.3 \mathrm{mmol}$, 1.0 eq.). A $5.0 \mathrm{M}$ solution of borane dimethylsulfide complex in diethylether (10.8 mmol, 2.5 eq.) was added slowly to the stirred reaction mixture at room temperature. Then the reaction mixture was heated under reflux for 4 hours. The reaction mixture was cooled to $0^{\circ} \mathrm{C}$, and methanol was slowly added to decompose the unreacted borane complex. The organic solvent was evaporated under reduced pressure. Purification by silica gel column chromatography $\left(\mathrm{NH}_{4} \mathrm{OH} / \mathrm{MeOH} / \mathrm{CHCl}_{3}, 4: 20: 80\right)$ afforded $0.6 \mathrm{~g}(46 \%)$ of MB-005 as a pale-yellow oil [ ${ }^{1} \mathrm{H}$ NMR $\left(500 \mathrm{MHz}, \mathrm{CD}_{3} \mathrm{OD}\right) \delta 6.81(\mathrm{~s}$, $1 \mathrm{H}), 6.74(\mathrm{~s}, 1 \mathrm{H}), 6.67(\mathrm{~s}, 1 \mathrm{H}), 4.74(\mathrm{t}, J=6.4 \mathrm{~Hz}, 1 \mathrm{H}), 3.76(\mathrm{~d}, J=$ $6.1 \mathrm{~Hz}, 2 \mathrm{H}$ ), 3.01-2.90 (m, 2H), 2.85 (sept, $J=7.3 \mathrm{~Hz}, 1 \mathrm{H}$ ), 1.95 (q, $J=$ $6.8 \mathrm{~Hz}, 2 \mathrm{H}), 1.91-1.84(\mathrm{~m}, 2 \mathrm{H}), 1.82-1.68(\mathrm{~m}, 4 \mathrm{H}), 1.39-1.25(\mathrm{~m}, 3 \mathrm{H})$, $1.23(\mathrm{~d}, J=6.7 \mathrm{~Hz}, 6 \mathrm{H}), 1.15-1.04(\mathrm{~m}, 2 \mathrm{H}) ;{ }^{13} \mathrm{C} \mathrm{NMR}(125 \mathrm{MHz}$, $\left.\mathrm{CDCl}_{3}\right) \delta 159.63,150.60,146.42,116.21,111.88,108.74,75.67,73.54$, $40.65,39.50,38.00,34.42,30.14,26.71,26.00,24.17,24.10$; ESIHRMS: $m / z$ calculated for $\mathrm{C}_{19} \mathrm{H}_{32} \mathrm{NO}_{2}^{+}[\mathrm{M}+\mathrm{H}]^{+}: 306.24276$; found: 306.24576].

3-Amino-1-(3-isopropyl-5-((2-propylpentyl)oxy)phenyl)propan1-ol (MB-006). The same procedure was followed as described for MB-005. A 2.0 M solution of borane dimethylsulfide complex in diethylether (10.25 mmol, 2.5 eq.) was added to a solution of the nitrile $\mathbf{7 b}(1.3 \mathrm{~g}, 4.1 \mathrm{mmol}, 1.0 \mathrm{eq}$. $)$ in anhydrous THF $(10 \mathrm{ml})$. Purification by silica gel column chromatography $\left(\mathrm{NH}_{4} \mathrm{OH} /\right.$ $\left.\mathrm{MeOH} / \mathrm{CH}_{2} \mathrm{Cl}_{2}, 4: 10: 90\right)$ afforded $0.63 \mathrm{~g}(47 \%)$ of MB-006 as a pale-yellow oil [ ${ }^{1} \mathrm{H} \mathrm{NMR}\left(500 \mathrm{MHz}, \mathrm{CDCl}_{3}\right) \delta 6.79(\mathrm{~s}, 1 \mathrm{H}), 6.74(\mathrm{~s}, 1 \mathrm{H}), 6.65$ (s, $1 \mathrm{H}), 4.90(\mathrm{t}, J=6.2 \mathrm{~Hz}, 1 \mathrm{H}), 3.81(\mathrm{~d}, J=5.6 \mathrm{~Hz}, 2 \mathrm{H}), 3.23-3.14(\mathrm{~m}$, $1 \mathrm{H}), 3.12-3.01(\mathrm{~m}, 1 \mathrm{H}), 2.84$ (sept, $J=7.3 \mathrm{~Hz}, 1 \mathrm{H}), 1.94(\mathrm{q}, J=6.0 \mathrm{~Hz}$, $2 \mathrm{H}), 1.81-1.72(\mathrm{~m}, 1 \mathrm{H}), 1.49-1.27(\mathrm{~m}, 8 \mathrm{H}), 1.22(\mathrm{~d}, J=6.6 \mathrm{~Hz}, 6 \mathrm{H}), 0.90$ $(\mathrm{t}, J=6.9 \mathrm{~Hz}, 6 \mathrm{H}) ;{ }^{13} \mathrm{C} \mathrm{NMR}\left(125 \mathrm{MHz} \mathrm{CDCl}_{3}\right) \delta 159.74,150.68$, $145.86,116.17,111.91,108.93,74.99,70.89,40.09,38.01,37.80,34.42$, 33.88, 24.16, 24.11, 20.16, 14.61; ESI-HRMS: $\mathrm{m} / \mathrm{z}$ calculated for $\mathrm{C}_{20} \mathrm{H}_{36} \mathrm{NO}_{2}^{+}[\mathrm{M}+\mathrm{H}]^{+}$: 322.27406; found: 322.27402].

3-Amino-1-(3-isopropyl-5-((2,6,6-trimethylcyclohex-1-en-1yl)methoxy)phenyl)propan-1-ol (MB-007). An oven-dried 50-ml, twonecked, round-bottomed flask equipped with a magnetic stir bar was sealed under argon. It had with two rubber septa, one of which contained a needle adapter to an argon inlet. The flask was charged with anhydrous THF $(25 \mathrm{ml})$ and a $2.0 \mathrm{M}$ solution of $\mathrm{LiAlH}_{4}$ in THF $\left(3.68 \mu \mathrm{l}, 7.36 \mathrm{mmol}, 2.5\right.$ eq.). At $0^{\circ} \mathrm{C}$, a solution of nitrile $7 \mathbf{c}(1 \mathrm{~g}$, $2.9 \mathrm{mmol}, 1$ eq.) in anhydrous THF ( $6 \mathrm{ml})$ was then added dropwise to this reaction mixture. The resulting yellow mixture was stirred at $0^{\circ} \mathrm{C}$ for 1 hour. At $0^{\circ} \mathrm{C}$, the reaction was quenched by slow addition of $\mathrm{H}_{2} \mathrm{O}$ (366 $\mu \mathrm{l}$ ) (caution: with evolution of hydrogen gas) followed sequentially by $15 \%(\mathrm{w} / \mathrm{v})$ aqueous $\mathrm{NaOH}(366 \mu \mathrm{l})$ and $\mathrm{H}_{2} \mathrm{O}(1.1 \mathrm{ml})$. Then, the amorphous mixture was stirred for 10 minutes at room temperature. The resulting mixture was vacuum filtered and washed well with $\mathrm{CH}_{2} \mathrm{Cl}_{2}$. The filtrate was dried over $\mathrm{MgSO}_{4}$ and concentrated under reduced pressure (Caspi et al., 2004). Purification by flash chromatography on a silica gel column $\left(\mathrm{NH}_{4} \mathrm{OH} / \mathrm{MeOH} / \mathrm{CH}_{2} \mathrm{Cl}_{2}, 4: 10: 90 \rightarrow 6: 20: 80\right)$ afforded $0.6 \mathrm{~g}(60 \%)$ of MB-007 as a colorless oil [ ${ }^{1} \mathrm{H}$ NMR $(500 \mathrm{MHz}$, $\left.\mathrm{CDCl}_{3}\right) \delta 6.87-6.79(\mathrm{~m}, 2 \mathrm{H}), 6.73(\mathrm{t}, J=1.9 \mathrm{~Hz}, 1 \mathrm{H}), 4.92(\mathrm{dd}, J=8.8$, $3.2 \mathrm{~Hz}, 1 \mathrm{H}), 4.43(\mathrm{~s}, 2 \mathrm{H}), 3.19-3.10(\mathrm{~m}, 1 \mathrm{H}), 3.03-2.94(\mathrm{~m}, 1 \mathrm{H}), 2.87$ (sept, $J=6.9 \mathrm{~Hz}, 1 \mathrm{H}), 2.04(\mathrm{t}, J=6.3 \mathrm{~Hz}, 2 \mathrm{H}), 1.94-1.76(\mathrm{~m}, 2 \mathrm{H}), 1.71(\mathrm{~s}$, $3 \mathrm{H}), 1.68-1.61(\mathrm{~m}, 2 \mathrm{H}), 1.53-1.47(\mathrm{~m}, 2 \mathrm{H}), 1.24(\mathrm{~d}, J=6.9 \mathrm{~Hz}, 6 \mathrm{H}), 1.05$ $(\mathrm{s}, 6 \mathrm{H}) ;{ }^{13} \mathrm{C} \mathrm{NMR}\left(125 \mathrm{MHz}, \mathrm{CDCl}_{3}\right) \delta 159.55,150.35,146.26,135.56$, 133.31, 116.20, 112.18, 108.71, 76.77, 75.66, 64.34, 40.64, 39.53, 39.30, $34.29,34.13,32.88,28.46,24.03,23.96,19.86,19.32$; ESI-HRMS: $\mathrm{m} / z$ calculated for $\mathrm{C}_{22} \mathrm{H}_{36} \mathrm{NO}_{2}^{+}[\mathrm{M}+\mathrm{H}]^{+}: 346.27406$; found 346.27399].

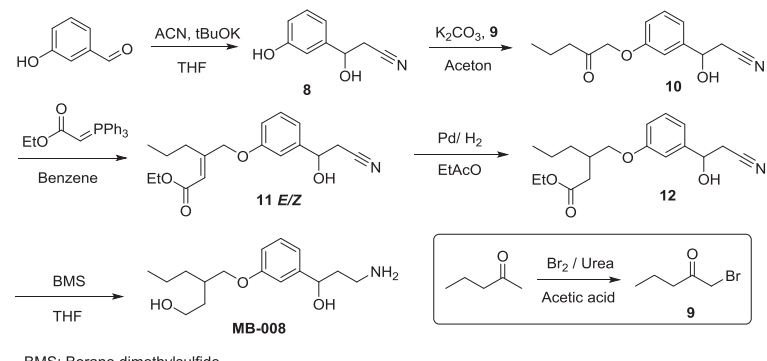

Scheme 4. Reagents and conditions for the synthesis of MB-008.

3-Hydroxy-3-(3-hydroxyphenyl)propanenitrile (8). An ovendried 100-ml, two-necked, round-bottomed flask was sealed under argon. The flask was charged with anhydrous THF (11 ml) and anhydrous acetonitrile $(465 \mu \mathrm{l}, 8.3 \mathrm{mmol})$, cooled to $-42^{\circ} \mathrm{C}$, and a solution of potassium tert-butoxide (2.06 g, $18.4 \mathrm{mmol})$ in THF $(20 \mathrm{ml})$ was added dropwise. After stirring for 45 minutes at $-42^{\circ} \mathrm{C}, 3$-hydroxy-benzaldehyde $(1 \mathrm{~g}, 8.2 \mathrm{mmol})$ in THF $(4.5 \mathrm{ml})$ was added dropwise. The reaction was allowed to warm to $-15^{\circ} \mathrm{C}$ over a 4 -hour period, and was then quenched by the slow addition of $25 \% \mathrm{NH}_{4} \mathrm{Cl}$ (aqueous) (14.5 ml). The layers were partitioned, and the aqueous phase was extracted twice with EtOAc. The combined organic layers were washed twice with $\mathrm{H}_{2} \mathrm{O}$ and once with brine, dried over $\mathrm{MgSO}_{4}$, and evaporated under reduced pressure. Purification by silica gel column chromatography (EtOAc/hexanes, 50:50) afforded $1.06 \mathrm{~g}$ $(80 \%)$ of compound 8 as a pale-yellow oil [ ${ }^{1} \mathrm{H} \mathrm{NMR}\left(400 \mathrm{MHz}, \mathrm{CDCl}_{3}\right) \delta 7.26$ $(\mathrm{t}, 1 \mathrm{H}, J=8 \mathrm{~Hz}), 6.95-6.91(\mathrm{~m}, 2 \mathrm{H}), 6.82(\mathrm{ddd}, 1 \mathrm{H}, J=1.2,2.4,8.0 \mathrm{~Hz}), 5.01$ $(\mathrm{t}, 1 \mathrm{H}, J=6.4 \mathrm{~Hz}), 2.77(\mathrm{~d}, 2 \mathrm{H}, J=6 \mathrm{~Hz}) \mathrm{ppm} ;{ }^{1} \mathrm{H}$ NMR values matched those in the literature (Caspi et al., 2004); $\left.{ }^{13} \mathrm{C} \mathrm{NMR} \mathrm{(100} \mathrm{MHz,} \mathrm{CDCl}_{3}\right) \delta$ $156.5,143.0,130.4,117.7,117.4,116.0,112.5,68.1,25.7 \mathrm{ppm}]$. 
1-Bromopentan-2-one (9). An oven-dried 100-ml, two-necked, round-bottomed flask was charged with urea $(9.8 \mathrm{~g}, 163.2 \mathrm{mmol}, 1.63$ eq.), acetic acid (39 ml), and 2-pentanone (10.65 ml, $100 \mathrm{mmol}, 1$ eq.). At $0^{\circ} \mathrm{C}, \mathrm{Br}_{2}(5.46 \mathrm{ml}, 17.05 \mathrm{~g}, 107.2 \mathrm{mmol}, 1.07 \mathrm{eq}$.) was added to the mixture and stirred at $18-20^{\circ} \mathrm{C}$ until the red color disappeared $(\sim 4.5$ hours). This mixture was then diluted with water $(150 \mathrm{ml})$ and extracted with $\mathrm{CH}_{2} \mathrm{CI}_{2}$. The extract was washed with saturated aqueous sodium carbonate, dried over $\mathrm{MgSO}_{4}$, and concentrated under reduced pressure (caution: compound is a lachrymator and needs to be handled carefully). Purification by silica gel column chromatography $\left(\mathrm{Et}_{2} \mathrm{O} /\right.$ hexanes, 3:97) afforded $4.2 \mathrm{~g}$ (26\%) of 1-bromopentan-2-one 9 as a-pale yellow liquid (Zav'yalov et al., 1989) $\left[{ }^{1} \mathrm{H}\right.$ NMR $(500 \mathrm{MHz}$, $\left.\mathrm{CDCl}_{3}\right) \delta 3.90(\mathrm{~s}, 2 \mathrm{H}), 2.66(\mathrm{t}, J=7.2 \mathrm{~Hz}, 2 \mathrm{H}), 1.67(\mathrm{~h}, J=7.5 \mathrm{~Hz}, 2 \mathrm{H})$, 0.96 (t, $J=7.2 \mathrm{~Hz}, 3 \mathrm{H}) ;{ }^{13} \mathrm{C} \mathrm{NMR}\left(125 \mathrm{MHz} \mathrm{CDCl}_{3}\right) \delta 202.25,41.85$, $34.44,17.50,13.72]$.

3-Hydroxy-3-(3-((2-oxopentyl)oxy)phenyl)propanenitrile (10). An oven-dried 100-ml, two-necked, round-bottomed flask equipped with a stir bar was sealed under argon. The flask was charged with the substituted phenol 8 (566 mg, $3.5 \mathrm{mmol}, 1.1$ eq.), 1-bromo-2-pentanone $\mathbf{9}(520 \mathrm{mg}, 3.2 \mathrm{mmol}, 1$ eq. $)$, anhydrous potassium carbonate $(646.8 \mathrm{mg}$, $4.68 \mathrm{mmol}, 1.3 \mathrm{eq}$.), and dry acetone $(32 \mathrm{ml})$. The reaction mixture was refluxed for 24 hours under argon atmosphere. The resulting slurry was cooled down, treated with water, and extracted with ether $(3 \times)$. The combined ether extracts were washed with $2.5 \mathrm{M} \mathrm{NaOH}$ aqueous solution and brine, dried over $\mathrm{MgSO}_{4}$, and evaporated under reduced pressure. Purification by silica gel column chromatography (EtOAc/ hexanes, 50:50) afforded $570 \mathrm{mg}(73 \%)$ of compound $\mathbf{1 0}$ as a paleyellow oil (White and Norcross, 1961) $\left[{ }^{1} \mathrm{H}\right.$ NMR $\left(500 \mathrm{MHz} \mathrm{CDCl}_{3}\right) \delta$ $7.32(\mathrm{t}, J=7.8 \mathrm{~Hz}, 1 \mathrm{H}), 7.01(\mathrm{~d}, J=7.2 \mathrm{~Hz}, 1 \mathrm{H}), 6.97(\mathrm{~s}, 1 \mathrm{H}), 6.85$ (dd, $J=8.2,2.6 \mathrm{~Hz}, 1 \mathrm{H}), 5.03(\mathrm{t}, J=6.2 \mathrm{~Hz}, 1 \mathrm{H}), 4.57(\mathrm{~s}, 2 \mathrm{H}), 2.76(\mathrm{~d}, J=$ $6.1 \mathrm{~Hz}, 2 \mathrm{H}), 2.56(\mathrm{t}, J=7.3 \mathrm{~Hz}, 2 \mathrm{H}), 1.67(\mathrm{~h}, J=7.7 \mathrm{~Hz}, 2 \mathrm{H}), 0.95$ $(\mathrm{t}, J=7.7 \mathrm{~Hz}, 3 \mathrm{H}) ;{ }^{13} \mathrm{C} \mathrm{NMR}\left(125 \mathrm{MHz}, \mathrm{CDCl}_{3}\right) \delta 207.10,158.28$, $142.91,130.29,118.70,117.05,114.68,111.94,72.74,69.94,40.96$, $27.95,16.69,13.73]$.

Ethyl-3-((3-(2-cyano-1-hydroxyethyl)phenoxy)methyl)hex-2enoate (11). An oven-dried 100-ml, two-necked, round-bottomed flask equipped with a magnetic stir bar was sealed under argon. The flask was charged with compound $10(389.0 \mathrm{mg}, 1.57 \mathrm{mmol}$, 1 eq.) and anhydrous benzene $(40 \mathrm{ml})$, followed by the addition of (ethoxycarbonylmethylene)triphenyl phosphorane $(75.9 \mathrm{mg}, 0.218 \mathrm{mmol}$, 1.09 eq.). This reaction mixture was refluxed under argon for 8 hours at $84^{\circ} \mathrm{C}$. Then, the solution was cooled down to room temperature and concentrated under reduced pressure. Purification by silica gel column chromatography (EtOAc/hexanes, 35:65) afforded $274 \mathrm{mg}(55 \%)$ of $E / Z$ mixture of compound $\mathbf{1 1}$ as a colorless oil. This was used for the next step without further characterization.

Ethyl 3-((3-(2-cyano-1-hydroxyethyl)phenoxy)methyl)hexanoate (12). An oven-dried 25-ml, two-necked, round-bottomed flask equipped with a magnetic stir bar was sealed under argon. The flask was charged with EtOAc (12 ml) and compound 11 (223 mg, $0.7 \mathrm{mmol}$, 1 eq.). Palladium on carbon ( $75 \mathrm{mg}, 10 \%$ wt., $0.07 \mathrm{mmol}, 0.1$ eq.) was added to the stirred mixture. The flask was purged under mild vacuum and backfilled three times with hydrogen. Hydrogenation was carried out via two-ply balloon pressure overnight at $25^{\circ} \mathrm{C}$. Then, the reaction mixture was filtered through a pad of celite, and the filtrate was concentrated under reduced pressure. Purification by silica gel column chromatography (EtOAc/hexanes, 30:70) afforded $200 \mathrm{mg}$ (89\%) of compound $\mathbf{1 2}$ as a colorless oil (Kimura et al., 2002) $\left[{ }^{1} \mathrm{H}\right.$ NMR $\left(500 \mathrm{MHz}, \mathrm{CDCl}_{3}\right) \delta 7.28(\mathrm{t}, J=7.8 \mathrm{~Hz}, 1 \mathrm{H})$, $6.94(\mathrm{~s}, 2 \mathrm{H}), 6.85(\mathrm{dd}, J=8.3,2.5 \mathrm{~Hz}, 1 \mathrm{H}), 4.99(\mathrm{t}, J=6.2 \mathrm{~Hz}, 1 \mathrm{H})$, $4.12(\mathrm{q}, J=7.2 \mathrm{~Hz}, 2 \mathrm{H}), 3.96-3.91(\mathrm{~m}, 1 \mathrm{H}), 3.90-3.84(\mathrm{~m}, 1 \mathrm{H}), 2.75(\mathrm{~d}$, $J=5.5 \mathrm{~Hz}, 2 \mathrm{H}), 2.63(\mathrm{brs}, 1 \mathrm{H}), 2.52-2.44(\mathrm{~m}, 1 \mathrm{H}), 2.41-2.31(\mathrm{~m}, 2 \mathrm{H})$, $1.55-1.45(\mathrm{~m}, 1 \mathrm{H}), 1.41-1.34(\mathrm{~m}, 3 \mathrm{H}), 1.24(\mathrm{t}, J=7.1 \mathrm{~Hz}, 3 \mathrm{H}), 0.92$ $(\mathrm{t}, J=6.8 \mathrm{~Hz}, 3 \mathrm{H}) ;{ }^{13} \mathrm{C} \mathrm{NMR}\left(125 \mathrm{MHz} \mathrm{CDCl}_{3}\right) \delta 173.07,159.44$, $142.70,129.98,117.70,117.29,114.75,111.62$, 70.18, 70.06, 60.41, $36.66,35.07,33.53,27.91,20.01,14.24,14.22]$.

3-((3-(3-Amino-1-hydroxypropyl)phenoxy)methyl)hexan-1-ol (MB-008). An oven-dried 10-ml, two-necked, round-bottomed flask was sealed under argon. The flask was charged with anhydrous THF (5 ml) and compound 12 (40 mg, $0.125 \mathrm{mmol}, 1$ eq.). Borane methylsulfide complex $5 \mathrm{M}$ in ether (125 $\mu \mathrm{l}, 0.626 \mathrm{mmol}, 5$ eq.) was slowly added to the stirred reaction mixture at room temperature. After complete addition, the reaction mixture was refluxed (2-4 hours) and then cooled to $0^{\circ} \mathrm{C}$. The unreacted borane complex was decomposed via addition of methanol (Brown et al., 1982). The organic solvent was evaporated under reduced pressure, and purification of the residue by silica gel column chromatography $\left(\mathrm{NH}_{4} \mathrm{OH} / \mathrm{MeOH} / \mathrm{CH}_{2} \mathrm{Cl}_{2}\right.$, 10:20:80) afforded $20.5 \mathrm{mg}$ (90\%) of MB-008 (a mixture of four stereoisomers) as a pale-yellow oil $\left[{ }^{1} \mathrm{H} \mathrm{NMR}\left(500 \mathrm{MHz}, \mathrm{CD}_{3} \mathrm{OD}\right) \delta\right.$ $7.22(\mathrm{t}, J=7.9 \mathrm{~Hz}, 1 \mathrm{H}), 6.97-6.88(\mathrm{~m}, 2 \mathrm{H}), 6.80(\mathrm{dd}, J=8.2,2.4 \mathrm{~Hz}$, $1 \mathrm{H}), 4.71(\mathrm{dd}, J=7.9,5.3 \mathrm{~Hz}, 1 \mathrm{H}), 3.90(\mathrm{~d}, J=5.4 \mathrm{~Hz}, 2 \mathrm{H}), 3.66(\mathrm{t}, J=$ $6.9 \mathrm{~Hz}, 2 \mathrm{H}), 2.84-2.71(\mathrm{~m}, 2 \mathrm{H}), 1.96(\mathrm{~h}, J=5.6 \mathrm{~Hz}, 1 \mathrm{H}), 1.92-1.81(\mathrm{~m}$, $2 \mathrm{H}), 1.77-1.59(\mathrm{~m}, 2 \mathrm{H}), 1.55-1.35(\mathrm{~m}, 4 \mathrm{H}), 0.94(\mathrm{t}, J=6.9 \mathrm{~Hz}, 3 \mathrm{H}) ;{ }^{13} \mathrm{C}$ NMR $\left(125 \mathrm{MHz}, \mathrm{CD}_{3} \mathrm{OD}\right) \delta 160.85,147.98,130.36,119.08,114.29$, $112.98,73.44,71.68,61.04,41.97,39.50,36.03,35.67,35.07,21.05$, 14.73; ESI-HRMS: $m / z$ calculated for $\mathrm{C}_{16} \mathrm{H}_{28} \mathrm{NO}_{3}^{+}[\mathrm{M}+\mathrm{H}]^{+}: 282.20637$; found 282.20633].

Retinoid Isomerase Activity Assays. Bovine RPE microsomes were prepared as previously described to provide the isomerase for the assay (Stecher et al., 1999). Synthesized primary amine ( $1 \mu \mathrm{l}$ in DMF, with a final concentration ranging between $50 \mathrm{nM}$ and $50 \mu \mathrm{M}$ ) was added to $10 \mathrm{mM}$ Bis-Tris propane/HCl buffer, $\mathrm{pH} 7.4$, containing $150 \mu \mathrm{g}$ RPE microsomes, $1 \%$ bovine serum albumin, $1 \mathrm{mM}$ disodium pyrophosphate, and $20 \mu \mathrm{M}$ cellular retinaldehyde-binding protein. The resulting mixture was preincubated at room temperature for 5 minutes. Then, all-trans-retinol $(1 \mu \mathrm{l}$ in DMF at a final concentration of $20 \mu \mathrm{M}$ ) was added. The resulting mixture was incubated at $37^{\circ} \mathrm{C}$ for 1 hour. The reaction was quenched by adding $300 \mu \mathrm{l}$ of methanol, and products were extracted with $300 \mu \mathrm{l}$ of hexanes. Production of 11-cisretinol was quantified by normal-phase high-performance liquid chromatography (HPLC) with $10 \%(\mathrm{v} / \mathrm{v})$ EtOAc in hexanes as the eluent at a flow rate of $1.4 \mathrm{ml} / \mathrm{min}$. Retinoids were detected by monitoring their absorbance at $325 \mathrm{~nm}$ and quantified based on a standard curve representing the relationship between the amount of synthetic 11-cis-retinol standard and the area under the corresponding chromatographic peak.

RPE65 Purification and Crystallization. RPE65 was purified from bovine RPE microsomes using the detergent $\mathrm{C}_{8} \mathrm{E}_{6}$ (Anatrace, Maumee, $\mathrm{OH}$ ) as previously described (Kiser et al., 2009). Inhibitors were added to the protein sample at the time of detergent solubilization as well as after concentrating the purified protein to a final concentration of $1 \mu \mathrm{M}$. Inhibitors were delivered to the samples as $100 \mathrm{mM}$ dimethylsulfoxide stock solutions. Crystallization was accomplished by mixing 1-2 $\mu \mathrm{l}$ of the concentrated protein sample with 1-2 $\mu \mathrm{l}$ of crystallization solution consisting of $40 \%(\mathrm{v} / \mathrm{v})$ polyethylene glycol 300, $100 \mathrm{mM}$ 2-(cyclohexylamino)ethanesulfonic acid- $\mathrm{NaOH}$ $(\mathrm{pH} \mathrm{9.5)}$, and $200 \mathrm{mM} \mathrm{NaCl}$ or $30 \%(\mathrm{v} / \mathrm{v})$ polyethylene glycol 400, $100 \mathrm{mM}$ 3-(cyclohexylamino)propanesulfonic acid- $\mathrm{NaOH}$ (pH 10.5), $500 \mathrm{mM}\left(\mathrm{NH}_{4}\right)_{2} \mathrm{SO}_{4}$, and $10 \%$ (v/v) glycerol (conditions \#26 and \#27, respectively of the Wizard Cryo screen; Rigaku, The Woodlands, TX). These conditions reliably yield untwinned hexagonal RPE65 crystals in which residues constituting the active-site cavity were previously shown to adopt active-like conformations in the presence of emixustatrelated molecules (Kiser et al., 2015). Crystals appeared in 1 or 2 days and grew to maximal dimensions of $75 \times 75 \times 300 \mu \mathrm{m}$ within 1 month. Mature crystals were harvested with Microloops (Mitegen, Ithaca, NY), flash cooled, and stored in liquid nitrogen prior to X-ray exposure.

Collection and Processing of X-ray Diffraction Data and Structure Refinement. X-ray diffraction data sets were collected at the NE-CAT ID-E beamline at the Advanced Photon Source (Lemont, IL). Diffraction data were indexed, integrated, and scaled with XDS (Kabsch, 2010). Data from three separate crystals of the RPE65/MB-004 complex were merged using XSCALE (Kabsch, 2010) to boost the signal-to-noise ratio and extend the high resolution limit. Initial models were generated through rigid body refinement of a previously reported RPE65 crystal structure (Protein Data Bank accession code 
4RYY) using the program REFMAC (Murshudov et al., 2011). Ligand and water atoms were removed from the model prior to conducting the initial refinement. The model was then completed and optimized by multiple rounds of manual real-space model building informed by MolProbity (Chen et al., 2010) close contact and geometry analyses in COOT (Emsley et al., 2010) followed by restrained reciprocal space refinement in REFMAC. Atomic coordinates and geometric restraint files for ligands were generated with the Grade Web Server. Some bond angle restraint weights were manually adjusted to ensure ideal ligand stereochemistry. Residues 109-126 and 197-201 in the RPE65/MB-004 complex and 110-126, 197-201, and 267-271 in the RPE65/MB-008 complex were excluded from the final models owing to their poorly resolved electron densities. The soundness of the final models was assessed with the MolProbity (Chen et al., 2015) and Worldwide Protein Data Bank (Read et al., 2011) structure-validation servers.

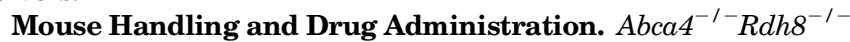
mice on a C57BL/6J background were generated as previously described (Maeda et al., 2009a). Male and female wild-type mice with C57BL/6J backgrounds were obtained from The Jackson Laboratory (Bar Harbor, $\mathrm{ME}$ ). $A b c a 4^{-1-} R d h 8^{-1-}$ mice used in this study were homozygous for the Leu450 allele of Rpe65 as determined by a genotyping protocol published previously (Grimm et al., 2004) and free of $\mathrm{Crb1/rd8}$ and $r d / r d$ mutations. Animals were housed either in the animal facility at the Case Western Reserve University School of Medicine, where they received a standard chow diet (LabDiet 5053; LabDiet, St. Louis, MO) and were maintained under a standard 12-hour light ( 300 lux) and 12-hour dark cycle, or at the Cole Eye Institute animal facility [animals used for electroretinogram (ERG) experiments], where they received LabDiet 2918 and were maintained under the standard 14-hour light ( 100 lux) and 10-hour dark cycle. Mice were dark-adapted overnight prior to all experiments. A cohort of evenly divided male and female $R d h 8^{-/-} \mathrm{Abca4^{-/- }}$ or C57BL/6J mice was used when they reached the age of 1-4 months. All tested primary amines were suspended in $100 \mu \mathrm{l}$ of soybean oil with less than $10 \%$ (v/v) dimethylsulfoxide and administered by oral gavage with a 22-gauge feeding needle. Experimental manipulations in the dark were done under dim red light transmitted through a Kodak No. 1 Safelight filter (transmittance $>560 \mathrm{~nm}$ ) (Kodak, Rochester, NY).

Induction of Acute Retinal Degeneration in $\mathrm{Abca4}^{-/-} \mathrm{Rdh}^{-/-}$ Mice. Following vehicle or test compound administration by oral gavage, 1- to 2-month-old $A b c a 4^{-1-} R d h 8^{-1-}$ mice were dark-adapted for 24 hours. Following induction of mydriasis with $1 \%$ tropicamide, the mice were exposed to fluorescent light (10,000 lux, 150-W spiral lamp; Commercial Electric, Cleveland, $\mathrm{OH}$ ) for 30 minutes in a white paper bucket, a treatment previously shown to generate retinal damage in the absence of protective agents (Zhang et al., 2015). Mice were then kept in the dark for an additional 3 days before examination of their retinal structure, which was carried out with ultrahigh-resolution spectral domain-optical coherence tomography (Bioptigen, Morrisville, NC) as previously reported (Zhang et al., 2015).

Identification of Retinaldehyde Conjugates with MB Compounds in $\boldsymbol{A b c a 4}^{-/-} \boldsymbol{R d h}^{-/-}$Mice. MB-005 or MB-006 (1 mg/mouse) was administrated by oral gavage to 1 - to 2 -month-old $A b c a 4^{-1-} R d h 8^{-/-}$ mice 24 hours prior to light exposure. After exposure to fluorescent light for 10 minutes, animals were euthanized, and their eyes were collected and homogenized in acetonitrile. After centrifugation, the supernatant was concentrated to $400 \mu \mathrm{l}$ under vacuum. Extracts $(100 \mu \mathrm{l})$ were injected into an LXQ linear ion trap mass spectrometer (Thermo Scientific, Waltham, MA) coupled to an Agilent 1100 HPLC system (Agilent Technologies, Santa Clara, CA). Separation of retinylidene Schiff bases was achieved on a reverse-phase C18 Phenomenex (Torrance, CA) HPLC column $(250 \times 4.60 \mathrm{~mm} ; 5 \mu \mathrm{m})$ by a linear gradient of acetonitrile in water of $50-100 \%$ within 15 minutes at a flow rate of $1.5 \mathrm{ml} / \mathrm{min}$. All solvents contained $0.1 \%$ formic acid $(\mathrm{v} / \mathrm{v})$. The HPLC effluent was directed into the mass spectrometer via an atmospheric pressure chemical ionization interface operated in the positive ionization mode. Identification of a retinylidene Schiff base was achieved by comparing its mass spectrum with that of an authentic standard.

Quantification of MB-005 in Mouse Eyes. MB-005 ( $1 \mathrm{mg} / \mathrm{mouse})$ was administrated by oral gavage to 1 - to 2-month-old C57BL/6J mice. The mice were then placed back in their home cages and kept in standard lighting conditions for either 6 or 24 hours. Then, mice were euthanized, and their eyes were homogenized in $1 \mathrm{ml}$ of $10 \mathrm{mM}$ sodium phosphate buffer, $\mathrm{pH} 7.4$, containing $50 \%$ methanol (v/v). The resulting mixture was extracted with $4 \mathrm{ml}$ of EtOAc. Extracts were dried in vacuo and reconstituted in $400 \mu \mathrm{l}$ of ethanol containing $1 \mathrm{nmol}$ emixustat as the internal standard. This solution $(100 \mu \mathrm{l})$ was analyzed by liquid chromatography-electrospray ionization-tandem mass spectrometry $(4.6 \times 150 \mathrm{~mm}$; Agilent ZORBAX Eclipse XDB-C18) with a gradient of acetonitrile in water (0-100\% over 30 minutes) as the eluent at a flow rate of $0.5 \mathrm{ml} / \mathrm{min}$. Amounts of amines were quantified based on a standard curve representing the relationship between the amount of MB-005 and the ratio of corresponding peak areas of MB-005 to emixustat.

Visual Chromophore Recovery Assay. C57BL/6J mice (1-2 months of age) were administered test compounds or vehicle control by oral gavage and then allowed to dark-adapt for 24 hours. Following induction of mydriasis with $1 \%$ tropicamide, the mice were exposed to bright light resulting in photoactivation of $>90 \%$ of rhodopsin and then kept in the dark for 6 hours to allow visual pigment regeneration. Afterward, animals were euthanized, and their eyes were collected and homogenized in $10 \mathrm{mM}$ sodium phosphate buffer, $\mathrm{pH} 7.4$, containing $50 \%$ methanol (v/v) and $40 \mathrm{mM}$ hydroxylamine. The resulting mixtures were extracted with $4 \mathrm{ml}$ of hexanes. Extracts were dried in vacuo, reconstituted in $300 \mu \mathrm{l}$ of hexanes, and $100 \mu \mathrm{l}$ of the extracts were injected into an HPLC system for analysis with $10 \%(\mathrm{v} / \mathrm{v})$ EtOAc in hexanes as the eluent. Amounts of 11-cis-retinal were quantified based on a standard curve representing the relationship between the amount of 11-cis-retinyl oxime and the area under the corresponding chromatographic peak.

ERG Recordings. C57BL/6J mice aged 1.5-4 months were anesthetized with ketamine $(80 \mathrm{mg} / \mathrm{kg})$ and xylazine $(16 \mathrm{mg} / \mathrm{kg})$. Mydriatic eye drops were used to dilate the pupil (1\% tropicamide; 2.5\% phenylephrine). ERGs were obtained with an active electrode that contacted the corneal surface; needle electrodes placed in the cheek and tail provided reference and ground leads, respectively. Strobe stimuli ranging from -3.6 to $1.2 \mathrm{log} \mathrm{cd} \mathrm{s} / \mathrm{m}^{2}$ were presented in an LKC (Gaithersburg, MD) ganzfeld. Responses were amplified, averaged, and stored with an LKC UTAS E-3000 signal averaging system.

Baseline dark-adapted ERGs were recorded from each mouse after overnight dark adaptation. After a minimum 1-day interval, MB compounds or vehicle were administered by gavage at approximately 9 a.m. The next morning, mydriatic eye drops were used to dilate the pupils, and mice were then exposed to a 10-minute 10,000-lux bleach. Mice were dark-adapted for 6 hours, after which their ERGs were recorded. The amplitude of the a-wave was measured from the prestimulus baseline to $8 \mathrm{~ms}$ after the stimulus flash. The amplitude of the b-wave was measured to the b-wave peak from either the a-wave trough or, if no a-wave was present, the baseline.

Study Approval. All animal procedures and experiments were approved by the Institutional Animal Care and Use Committees of Case Western Reserve University or the Cleveland Clinic, and conformed to recommendations of the American Veterinary Medical Association Panel on Euthanasia and the Association for Research in Vision and Ophthalmology.

\section{Results}

Identification of Potential Sites for Emixustat Modification Based on RPE65 Crystal Structures. The emixustat molecule can be divided into three basic regions: a polar aminopropanol moiety, a lipophilic cyclohexyl methoxy ring, and a central phenyl ring to which both substituents are 
attached (Fig. 1A). MB-001 differs from emixustat by the substitution of a trimethyl hexenyl ring in place of the cyclohexyl moiety. These molecules reside in the region of the RPE65 active-site cavity that is proximal to its membraneembedded entrance (Kiser et al., 2015). The aminopropanol moiety, located deepest within the binding pocket of the molecule, forms electrostatic interactions with Thr147 and Glu148 as well as with an iron-bound fatty acid carboxylate. Such interactions appear to contribute substantially to the binding affinity, making alterations to this part of the molecule that disrupt active-site binding affinity easily envisioned, such as by removal or alkylation of the hydroxyl moiety. However, because such changes could influence the reactivity of the amino group toward retinaldehyde, we chose not to attempt modifications of this region of the molecule. Notably, we previously showed that emixustat forms a retinaldehyde Schiff base more efficiently than retinylamine (Zhang et al., 2015), possibly due to the nearby hydroxyl moiety that can intramolecularly interact with the iminium functionality.

The central phenyl ring is involved in $\pi-\pi$ interactions with Phe103 as well as van der Waals contacts with a number of other active-site residues. Notably, the active-site region just above the phenyl ring is relatively polar, with two well ordered water molecules engaged in hydrogen-bonding interactions with its constituent residues. These water molecules are located $\sim 3.3 \AA$ away from C5 of the emixustat molecule (Fig. 1B). We reasoned that introduction of a bulky lipophilic group to the region of the phenyl ring close to this polar pocket could effectively prevent binding of the derivative molecule to the RPE65 active site as a result of steric and electrostatic repulsive forces (Fig. 1C).

The terminal aliphatic ring moieties of these molecules, located closest to the active-site entrance, are the most mobile regions of the molecule as evidenced by their higher average atomic displacement parameters and multiple conformations observed in different crystal forms (Kiser et al., 2015; Zhang et al., 2015). The long-range van der Waals interactions formed between this portion of the molecules and residues forming the active-site opening are expected to minimally contribute to the binding affinity of emixustat and MB-001. This region thus appeared to be a strong candidate for structural modification to improve active-site binding affinity. Two binding cavities are present on either side of the cyclohexyl moiety: one polar and filled with water molecules, and the other a small hydrophobic cavity formed by residues Ile259, Phe264, Tyr275, Cys278, and Phe279 that could serve as a site of interaction for suitable derivatives (Fig. 1B). In particular, we hypothesized that replacement of the terminal 6-membered ring with short linear moieties could result in molecules with the ability to engage these unexploited potential binding cavities (Fig. 1C).

Thus, we developed novel synthetic routes for the generation of two classes of compounds: one series in which the terminal 6-membered ring of emixustat/MB-001 was substituted by either heptan-4-yl (MB-004) or 1-hydroxyhexan-3-yl (MB-008) groups, and another in which an isopropyl moiety was added on the meta position of the phenyl ring with the terminal lipophilic
A<smiles>[R]COc1cccc(C(O)CCN)c1</smiles>

emixustat<smiles>CCCC(C)CCC</smiles>

(R)-MB-004

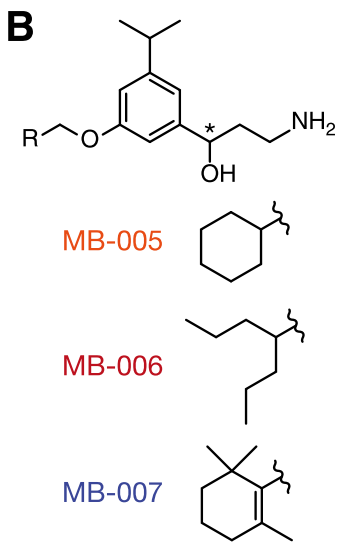

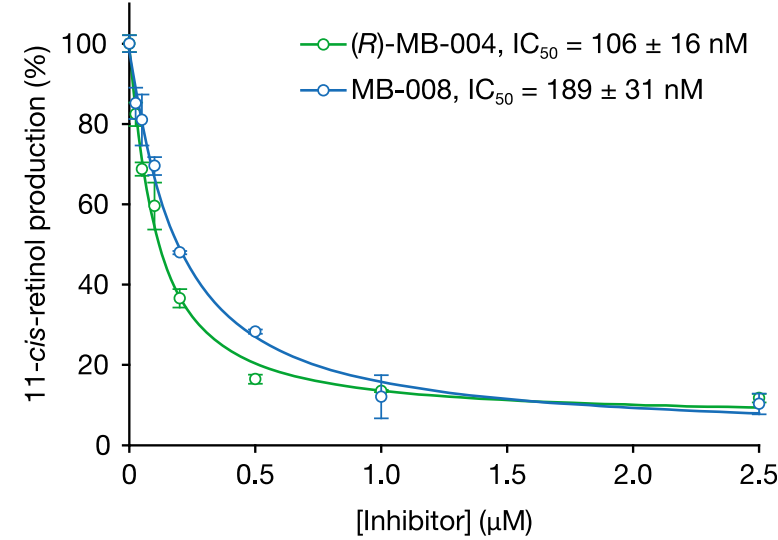

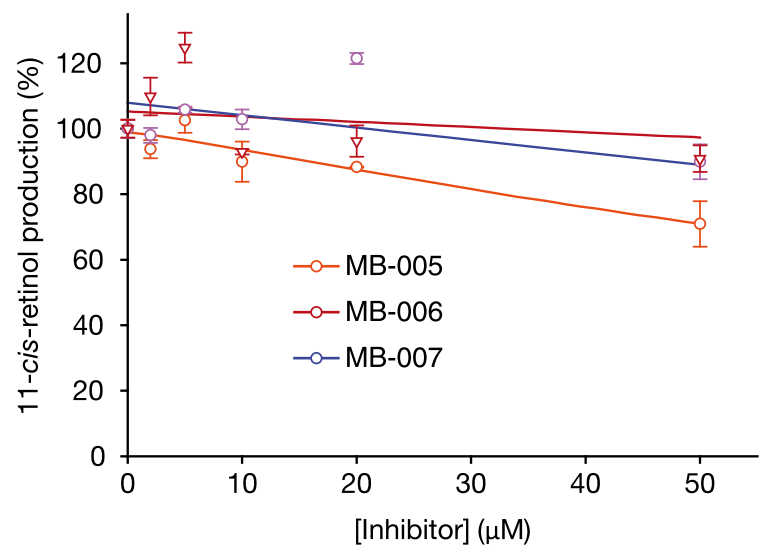

Fig. 2. In vitro inhibition of retinoid isomerase activity by MB compounds. Primary amines were preincubated with bovine RPE microsomes at room temperature for 5 minutes, then all-trans-retinol was added, and the mixtures were incubated at $37^{\circ} \mathrm{C}$. After 1 hour of incubation, all reactions were quenched by the addition of methanol. Inhibition of RPE65 enzymatic activity was measured as a decline in 11-cis-retinol production. (A) Production of 11-cis-retinol in the presence of amines designed to inhibit RPE65. The $\mathrm{IC}_{50}$ values for emixustat and $(R)$-MB-001 in this assay are $232 \pm 3$ and $396 \pm 58 \mathrm{nM}$, respectively (Kiser et al., 2015). (B) Production of 11-cis-retinol in the presence of amines with bulky groups that could hinder RPE65 active-site binding. Chiral centers are marked with asterisks. 
moiety consisting of either cyclohexyl (MB-005), heptan-4-yl (MB-006), or trimethyl hexenyl (MB-007) groups (Fig. 2, A and $\mathrm{B}$, left panels). Based on the structural features outlined earlier, we hypothesized that the latter group of compounds would exhibit impaired inhibitory activity toward RPE65, whereas the inhibitory activity of the former group could be enhanced owing to potentially reinforced binding interactions. Both sets of compounds contained unmodified primary amine functionalities and thus were anticipated to retain their retinaldehyde sequestration activity (Fig. 1C).

Changes to the Emixustat/MB-001 Core Structure Alter In Vitro Retinoid Isomerase Inhibitory Activity. To test these structural predictions, we examined the inhibitory activity of candidate compounds against RPE65 activity in an in vitro retinoid isomerase assay using bovine RPE microsomes as the enzyme source. Consistent with our hypothesis, both MB-004 and MB-008 exerted more potent RPE65 inhibitory effects relative to emixustat and MB-001. Compared with emixustat (Kiser et al., 2015), the two compounds MB-004 and MB-008 were about 2.2 and 1.2 times more potent, with $\mathrm{IC}_{50}$ values of $106 \pm 16$ and $189 \pm 31 \mathrm{nM}$, respectively (Fig. 2A). Because racemic forms of emixustat and MB-008 were used in these experiments, whereas MB-004 was synthesized as the pure $R$-isomer, stereochemical differences must be accounted for when comparing the potencies of these compounds. We previously reported that $(R)$-emixustat is $\sim 1.6$ times more potent than the $S$-isomer (Zhang et al., 2015). The estimated $\mathrm{IC}_{50}$ of $(R)$-emixustat in this assay is therefore around $175 \mathrm{nM}$, making MB-004 approximately 1.7 times more potent in this head-to-head comparison. The four possible isomeric forms of MB-008 make head-to-head potency comparisons to emixustat more difficult, but the racemic mixture is at least as potent as $(R)$-emixustat, and the possibility remains that one particular isomer could have even higher potency. Conversely, MB-005, MB-006, and MB-007 all exhibited little or no inhibition against retinoid isomerization in this assay up to concentrations of $50 \mu \mathrm{M}$, consistent with their inability to access the RPE65 active site and block retinyl ester uptake (Fig. 2B). Thus, these structure-guided modifications to the emixustat molecule modulated RPE65 inhibition in a manner consistent with our expectations.

MB-004 and MB-008 Engage Binding Sites in the RPE65 Active Site Cavity That Unused by Emixustat and MB-001. To test our hypothesis that binding of MB-004 and MB-008 to the RPE65 active site could be strengthened by interactions with cavities located near the cyclohexanyl ring of emixustat, we determined crystal structures of bovine RPE65 in complex with both molecules. The RPE65-MB-004 and RPE65-MB-008 structures, refined against data extending to 2.2- and 2.1-Å resolution, respectively, were obtained by cocrystallization, with the compounds present in an

TABLE 1

X-ray data and crystallographic refinement statistics

\begin{tabular}{|c|c|c|}
\hline \multicolumn{3}{|c|}{ Data collection and processing ${ }^{a}$} \\
\hline Crystal & MB- $004^{b}$ & MB-008 \\
\hline X-ray source & NECAT-24 ID-E & NECAT-24 ID-E \\
\hline Wavelength $(\AA)$ & 0.97919 & 0.97918 \\
\hline Unit cell parameters $(\AA)$ & $\mathrm{a}=175.73, \mathrm{c}=86.37$ & $\mathrm{a}=175.32, \mathrm{c}=86.65$ \\
\hline Space group & $P 6_{5}$ & $P 6_{5}$ \\
\hline Resolution $(\AA)$ & $50-2.20(2.26-2.20)$ & $50-2.10(2.23-2.10)$ \\
\hline Unique reflections & $77,111(5637)$ & $86,966(14,111)$ \\
\hline Multiplicity & $12.1(10.8)$ & $4.0(3.9)$ \\
\hline Completeness (\%) & $100(100)$ & $98(99)$ \\
\hline$<I / \sigma I>$ & $11.3(1.2)$ & $9.34(0.96)$ \\
\hline$R_{\text {meas }} I(\%)$ & $20.9(228.1)$ & $15.2(180.8)$ \\
\hline $\mathrm{CC}_{1 / 2}(\%)$ & $99.7(40.1)$ & $99.6(36.5)$ \\
\hline \multicolumn{3}{|c|}{ Refinement $^{c}$} \\
\hline Resolution $(\AA)$ & $47.9-2.2$ & $47.9-2.1$ \\
\hline No reflections & 73,171 & 82,511 \\
\hline$R_{\text {work }} / R_{\text {free }}(\%)$ & $17.0 / 20.9$ & 17.6/21.1 \\
\hline No atoms & 8894 & 8829 \\
\hline Protein & 8332 & 8187 \\
\hline \multirow{3}{*}{ Active-site ligand/ions } & Fe: 2 & Fe: 2 \\
\hline & MB-004: 40 & MB-008: 40 \\
\hline & Palmitate: 36 & Palmitate: 36 \\
\hline Water & 447 & 530 \\
\hline$<B$-factor $>\left(\AA^{2}\right)$ & 43.6 & 41 \\
\hline Protein & 43.5 & 41 \\
\hline \multirow{3}{*}{ Active-site ligand/ions } & Fe: 34 & Fe: 32 \\
\hline & MB-004: 41 & MB-008: 44 \\
\hline & Palmitate: 53 & Palmitate: 52 \\
\hline Water & 44 & 43 \\
\hline \multicolumn{3}{|l|}{ Root-mean-square (RMS) deviations } \\
\hline Bond lengths $(\AA)$ & 0.012 & 0.012 \\
\hline Bond angles $\left(^{\circ}\right)$ & 1.55 & 1.54 \\
\hline Ramachandran plot (\% favored/outliers) ${ }^{d}$ & $97.4 / 0$ & $98.5 / 0$ \\
\hline MolProbity score (percentile) & 100th & 100th \\
\hline PDB accession code & 5UL5 & 5ULG \\
\hline
\end{tabular}

PDB, Protein Data Bank; RMS, Root-mean-square.

${ }^{a}$ Diffraction data processing was carried out with XDS.

${ }^{b}$ Merged data from three crystals.

${ }^{c}$ Structure refinement was carried out with REFMAC.

${ }^{d}$ Assessed with MolProbity. 

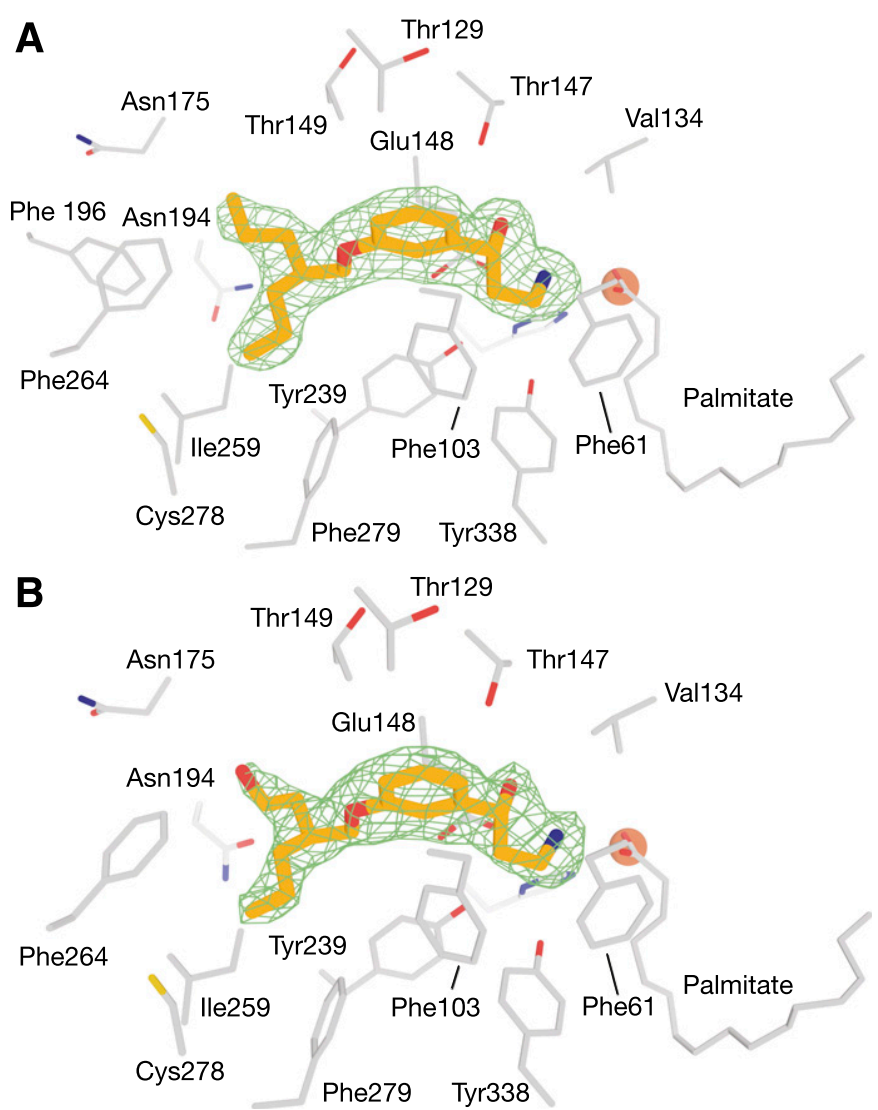

Fig. 3. Binding of MB-004 and MB-008 to the RPE65 active-site pocket. MB-004 (A) and MB-008 (B) are shown as orange sticks. Residues within $4.5 \AA$ of the MB ligands are shown as gray sticks. The iron cofactor and the iron-bound palmitate ligand are shown as brown spheres and gray sticks, respectively. The green mesh represents a $\sigma \mathrm{A}$-weighted $\mathrm{F}_{\mathrm{o}}-\mathrm{F}_{\mathrm{c}}$ electron density map calculated after deletion of the ligand and 30 cycles of REFMAC refinement. Residues within $4.5 \AA$ of the ligand were harmonically restrained to their initial positions during refinement. The electron density suggested that the $R, S$ form of MB-008, which was added to the protein as a racemic mixture, was the dominant isomer occupying the binding pocket.

approximately 5-fold molar excess over the protein under conditions similar to those used in prior RPE65-emixustat structure determinations (Table 1 ). In both cases, the ligands were clearly identified from their initial residual electron density maps (Fig. 3, A and B). The 3-amino-1-phenylpropan1-ol moieties, common to MB-004, MB-008, and emixustat, were found in essentially identical conformations in all structures (Fig. 4 ). The $(R)$-isomer of the 3-amino-1-hydroxypropylphenyl moiety of MB-008 appeared to be the dominant form of the molecule occupying the active site, in agreement with our previous study on the binding of emixustat isomers to the RPE65 active site (Zhang et al., 2015).

The dipropanyl tail of MB-004 adopted an extended conformation, with one half of the moiety occupying the aforementioned hydrophobic cavity in van der Waals contact with the side chains of residues Ile259, Phe264, Tyr275, and Phe279 (Fig. 4B). The other half of this moiety chain was also found in an extended conformation near the active-site tunnel entrance, but was less restrained, being held in place only by a few relatively less-favorable van der Waals contacts with the Glu148 backbone carbonyl oxygen, backbone atoms of Gly176, and a nearby water molecule. This difference in binding interactions was reflected in the $\sim 11 \%$ lower average $B$-factor observed for the chain occupying the hydrophobic cavity (44 $\AA^{2}$ ) compared with the other half of the substituent with sparser interaction partners $\left(49 \AA^{2}\right)$.

The 7-membered tail of MB-008 adopted a conformation very similar to that of MB-004 with the exception of a slight rotation of the hydroxyethyl moiety that allowed formation of a hydrogen bond with a water molecule located in the polar cavity mentioned earlier (Fig. 4C). Trial refinements with the molecule modeled in the four possible isomers suggested that the $R, S$ form of the molecule is the dominant form bound in the active site. Despite the apparently more-favorable interaction made by the hydroxyethyl side chain, its $B$-factors remained elevated compared with the rest of the molecule, similar to the analogous region of MB-004.

In summary, replacement of the emixustat cyclohexyl tail with a short linear alkane moiety appeared to strengthen binding affinity owing to the fulfillment of favorable van der Waals and hydrophobic interactions with a lipophilic pocket located near the active-site entrance. The presence of a second linear moiety with a capability to form hydrogen-bonding interactions with polar residues and solvent molecules located across from the lipophilic pocket

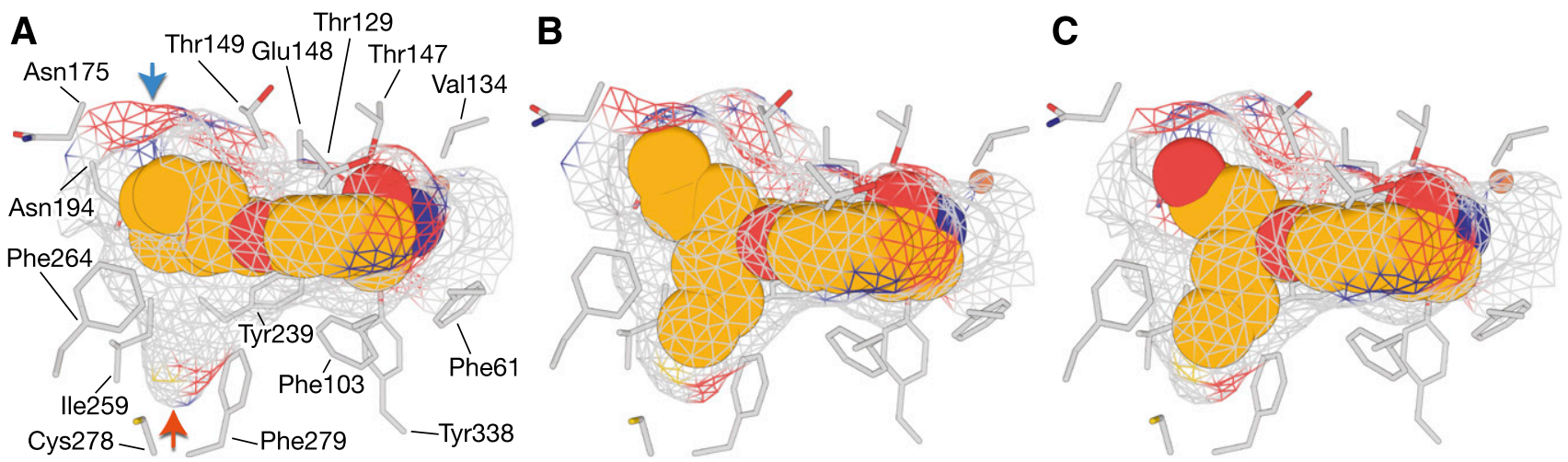

Fig. 4. Occupancy of MB ligands in the membrane-proximal binding pockets of the RPE65 cavity. Ligands are shown as van der Waals spheres with carbon, nitrogen, and oxygen atoms colored orange, blue, and red, respectively. (A) The cyclohexyl ring of emixustat resides close to a polar, water-filled pocket (blue arrow) within the membrane-proximal region of the active-site pocket. The bulkiness of the cyclohexyl moiety prevents it from occupying a nearby apolar pocket (red arrow) with the potential to promote ligand binding through hydrophobic and van der Waals forces. (B) One half of the MB-004 dipropyl moiety occupies the aforementioned hydrophobic cavity forming van der Waals contacts with residues lining the pocket, with the other half occupying a less constrained position near the active-site opening. (C) The binding pose for MB-008 is nearly identical to that of MB-004. Notably, the polar hydroxyl tail of MB-008 fails to form significant hydrogen bonding or dipolar interactions with residues or water molecules comprising the polar side of the proximal active-site pocket. 


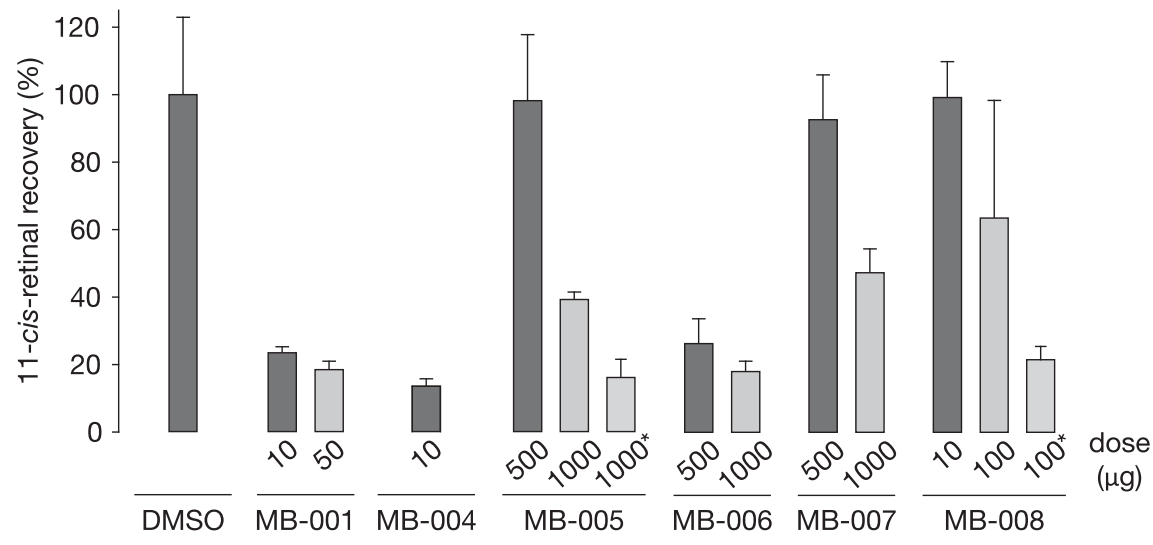

Fig. 5. Inhibition of visual chromophore regeneration in C57BL/6J mouse eyes by MB compounds. MB drugs were administrated to 1- to 2-month-old mice by oral gavage 24 or 4 hours (marked by asterisks) prior to light exposure for 10 minutes. Mice were then allowed to recover in darkness for 6 hours. The ocular 11-cis-retinal levels were measured by HPLC. Values are expressed as percentages of the control group (displayed as means \pm S.D.; $n=3$ for each group except the MB-008 group in which $n=9$ ). DMSO, dimethylsulfoxide.

appeared to contribute minimally to the binding affinity of MB-008.

Effects of MB Compounds on Visual Cycle Function In Vivo. Next, we tested the effects of the MB compound series on retinoid cycle function in living mice. For these experiments, mice were given a single dose of the test compound by oral gavage, and 24 hours later, they were subjected to light exposure that bleached $>90 \%$ of rhodopsin. After light treatment, mice were allowed to dark-adapt for 6 hours before their ocular retinoid content was determined by HPLC. MB-004, at a dose of $10 \mu \mathrm{g} / \mathrm{mouse}$, dramatically suppressed visual chromophore regeneration to at least the same degree as MB-001 at the same dose (Fig. 5). By contrast, mice treated with $10 \mu \mathrm{g}$ of MB-008 recovered visual chromophore to a level comparable to vehicle-treated mice, with a 10 -fold higher dose producing only a $30 \%$ suppression in visual cycle function, which was unexpected given the strong in vitro retinoid isomerase inhibitory effects of this compound (Fig. 2A). To examine whether the lack of MB-008 efficacy at 24 hours was due to insufficient concentrations resulting from a shorter elimination half-life or poorer bioavailability, we repeated the experiment with mice subjected to light exposure 4 hours after MB-008 administration. A 100- $\mu \mathrm{g}$ dose of MB-008 suppressed visual chromophore regeneration by $\sim 80 \%$, supporting the idea that the retinaldehyde

concentration of MB- 008 could be subeffective at the longer post-treatment light challenge.

Despite the near absence of in vitro retinoid isomerase inhibitory effects for MB-005, MB-006, and MB-007 (Fig. 2B), these compounds discernably slowed chromophore regeneration in living mice, albeit at doses much higher than those used for MB-001 (Fig. 5). Five-hundred-microgram doses of MB-005 and MB-007 had minimal effects on 11-cis-retinaldehyde recovery, whereas $\sim 50 \%$ inhibition was observed with both compounds at a 2 -fold higher dose. By contrast, 500 and $1000 \mu \mathrm{g}$ of MB-006 suppressed visual chromophore production to $\sim 30$ and $\sim 20 \%$ of the vehicle control, respectively. Similar to MB-008, in the case of MB-005, we found that shortening the time between drug administration and the light challenge enhanced the inhibitory effects of the treatment, suggesting that substantial MB-005 clearance occurred in the 24 hours following drug administration.

Impact of Visual Cycle Modulators on the Mouse ERG. We next examined the impact of these compounds on normal retinal function, as assessed by the ERG. Figure 6A presents a family of dark-adapted ERGs measured 6 hours after mice underwent a $95 \%$ bleach presented 1 day following gavage with vehicle or MB compounds at doses indicated as effective by retinoid analysis. Mice gavaged with vehicle alone had dark-adapted ERGs that were comparable in waveform to

A
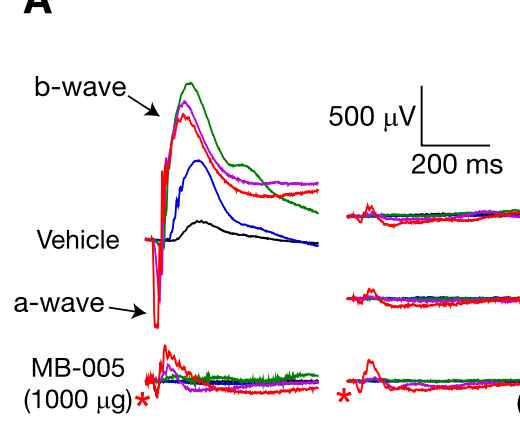

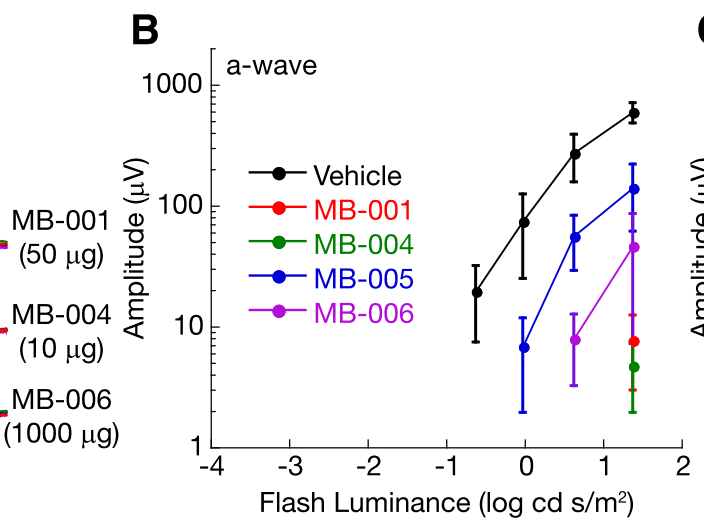

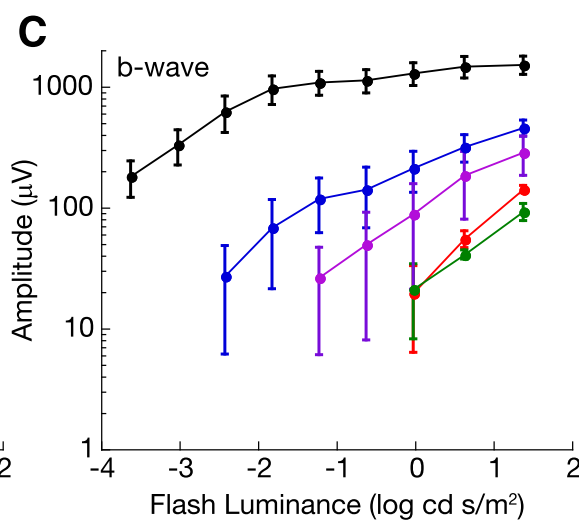

Fig. 6. ERG responses of C57BL/6J mice treated with visual cycle modulators. MB compounds or vehicle was administered by oral gavage to 1.5- to 4-month-old C57BL/6J mice 24 hours prior to 10-minute light exposure followed by 6 hours of dark adaptation. Dark-adapted ERGs were obtained using strobe flash stimuli. (A) Representative ERGs obtained from mice treated with vehicle or an MB compound at the indicated dose. Flash strength is colorcoded (black, $-3.6 \log \mathrm{cd} \mathrm{s} / \mathrm{m}^{2}$; blue, $-2.4 \log \mathrm{cd} \mathrm{s} / \mathrm{m}^{2}$; green, $-1.2 \log \mathrm{cd} \mathrm{s} / \mathrm{m}^{2} ;$ purple, $0.0 \log \mathrm{cd} \mathrm{s} / \mathrm{m}^{2} ;$ red, $1.2 \log \mathrm{cd} \mathrm{s} / \mathrm{m}^{2}$ ). Red asterisks next to the a-waves for MB-005 and MB-006 indicate responses larger than those obtained from MB-001 or MB-004. Amplitudes of the major components of the ERG are shown in (B) (a-wave) and (C) (b-wave). Data points indicate average ( \pm S.E.M.) from three to four mice per treatment group. 
those obtained in a pretreatment baseline study but were slightly smaller in amplitude. In comparison, mice administered MB compound molecules had decreased-amplitude ERGs indicative of incomplete bleaching recovery. For example, animals treated with MB-001 and MB-004 had responses to low-luminance stimuli that did not differ from background noise levels. In response to higher-luminance stimuli, reproducible responses were observed. That these responses reflected dark-adapted cone activity is supported by their similarity to responses obtained from transducin knockout mice which lack rod-mediated activity, while retaining normal cone-mediated responses and featuring a very similar ERG phenotype (Calvert et al., 2000; Wu et al., 2004). Mice treated with MB-005 and MB-006 had less-incomplete recovery, as evidenced by a small-amplitude electronegative a-wave to high luminance stimuli (red asterisk) and larger amplitude b-waves (Fig. 6, B and C).

Isopropyl Emixustat Derivatives Distribute into the Eye and Form Retinaldehyde Schiff Base Conjugates In Vivo. The unexpected ability of isopropyl emixustat derivatives to slow visual chromophore regeneration indicated that the ocular distribution of these compounds was preserved despite the structural modification. To confirm the ocular distribution of this compound series, we directly measured the quantity of MB-005 in the eye by mass spectrometry after a 1-mg dose. Using emixustat as an internal standard, we determined that the ocular level of MB-005 attained 6 hours after treatment was $\sim 140 \mathrm{pmol} / \mathrm{eye}$, and this declined only slightly at the 24-hour post-treatment time point. This minimal change in ocular MB-005 concentration contrasts with the $\sim 3$-fold greater inhibition of visual cycle function observed at 4 hours versus 24 hours for this compound. These results indicate that the isopropyl-derivative series compounds could persist in the eye for extended periods of time to exert their effects.

With the effects of these compounds on visual cycle function established, we next examined their ability to form retinaldehyde Schiff base conjugates in vivo. For these experiments, Abca4 ${ }^{-1-} R d h 8^{-/-}$mice, a model for Stargardt macular dystrophy that exhibits delayed all-trans-retinal clearance, were gavaged with MB-005 or MB-006 and then subjected to bright light exposure 6 hours later. Retinoids were extracted and analyzed by mass spectrometry. MB-005- and MB-006-retinal Schiff base adducts formed in vitro were prepared for use as standards. Acetonitrile extracts from the eyes of mice treated with either MB-005 or MB-006 contained compounds matching the expected molecular weight of the respective Schiff base adducts, with retention times (Fig. 7, A and B, left) and tandem mass spectra (Fig. 7, A and B, right) exactly matching those of the standards. These data demonstrate that emixustat derivatives with substitutions that alter their ability to directly inhibit RPE65 have a retained ability to form retinaldehyde Schiff base adducts in vivo, suggesting that they can exert protective effects against retinaldehyde toxicity.

Protective Effects of MB Compounds against Retinal Phototoxicity. To examine the ability of the MB compounds to protect against retinal phototoxicity, we administered the compounds to Abca4-I - Rdh8- / - mice by oral gavage, and after 24 hours, the mice were exposed to white light of sufficient intensity to cause retinal damage in the absence of treatment with protective agents. Three days later, retinas were imaged in vivo by optical coherence tomography, with preservation of outer nuclear layer thickness and light-scattering
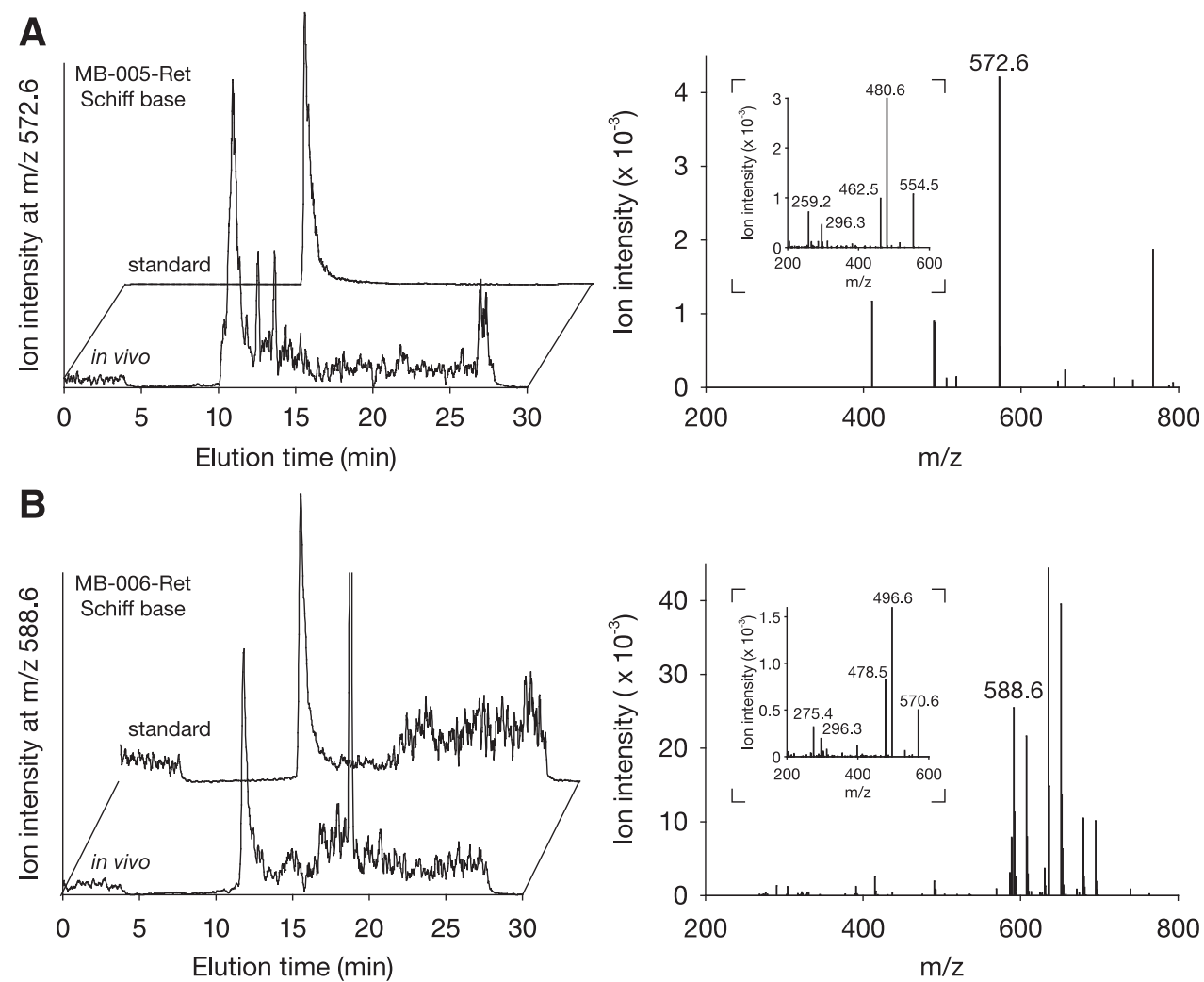

Fig. 7. Identification of retinal Schiff base conjugates in $\mathrm{Abca4^{-1- }} R d h 8^{-1-}$ mice treated with MB-005 and MB-006. Following drug administration, mice (1-2 months of age) were exposed to intense light for 10 minutes. After a 30-minute recovery under darkness, animals were sacrificed and retinoids were extracted from the eyes. (A and B) Shown in the left panels are selective ion chromatograms for MB-005-retinal conjugates (MB-005-Ret) $($ at $\mathrm{m} / \mathrm{z}=572.6)(\mathrm{A})$ and MB-006-retinal conjugates (MB-006-Ret) (at $\mathrm{m} / \mathrm{z}=588.6$ ) (B). Panels on the right show the mass spectra of the peaks coinciding with the retention times of their authentic standards. Insets display the tandem mass spectrometry fragmentation patterns of the two imines, which were identical to their corresponding standards. 
properties serving as markers for retinal health. MB-001, capable of preventing light-induced retinal degeneration through both direct RPE65 inhibition (Kiser et al., 2015) and retinaldehyde sequestration, served as a positive control for these experiments.

MB-004 at a dose about 2.5 times greater than that required for near complete suppression of in vivo visual cycle function $(25 \mu \mathrm{g})$ produced about an $80 \%$ protective effect relative to MB-001, which was fully protective at a dose of $50 \mu \mathrm{g}$ (Fig. 8). This finding, showing incomplete retinal protection in the face of profound visual cycle suppression, suggested an incomplete causal relationship between the two activities. In other words, the lack of full outer nuclear layer preservation observed for MB-004 supports the idea that retinal protection exerted by primary amine compounds is not fully explained by their degree of visual cycle inhibition. MB-008, which also directly inhibits RPE65, was poorly protective even at doses as high as $0.25 \mathrm{mg}$ when administered 24 hours prior to the light challenge. Consistent with our in vivo visual chromophore regeneration assay suggesting that MB-008 concentrations were lower compared with MB-004, we found that shortening the duration between compound administration and the light challenge from 24 to 6 hours substantially improved the protective effects of this compound. However, even after these shorter time periods, MB-008 could only exert full protection at a dose 10 times higher than that used for MB-004, which would be expected to near-maximally suppress visual cycle function.

The isopropyl MB compound series also exerted variable protective effects (Fig. 8). At one extreme, MB-007 was no more protective than vehicle control at the high dose of $1 \mathrm{mg}$ per mouse, even though this compound unexpectedly suppressed visual chromophore regeneration by $\sim 50 \%$ at the same dose (Fig. 5). MB-006 was protective at a dose of $1 \mathrm{mg}$ but not $0.5 \mathrm{mg}$ per mouse. Similar to MB-007, the 0.5-mg dose of MB-006 strongly suppressed visual cycle function, suggesting that such inhibition is irrelevant to the retinal protective effects of these compounds (Fig. 5). MB-005 was unique among this set of compounds for its ability to confer protection against retinal phototoxicity at doses that preserved substantial visual cycle function. At a dose of $0.5 \mathrm{mg} / \mathrm{mouse}$, which had no measurable effect on visual cycle function (Fig. 5), MB-005 treatment resulted in an $\sim 50 \%$ protective effect as compared with the MB-001 control (Fig. 8). Doubling the dose resulted in an 80\% protective effect, similar to a 25- $\mu$ g dose of MB-004 (Fig. 8), with about $60 \%$ suppression of visual chromophore regeneration activity (Fig. 5). A 2-mg/mouse dose of MB-005 fully protected the retina from light damage, although visual cycle function was also severely impaired at this dose (Fig. 5 and 8).

\section{Discussion}

In this study, we subjected visual cycle modulators with retinoid-like structures to chemical modifications designed to either block or potentiate inhibition of RPE65 with retained ability to sequester retinaldehyde via Schiff base formation. Preservation of the retinoid backbone was chosen on the basis that retinoids and retinoid-like molecules are subject to specialized ocular transport systems and enzymatic machinery, including the RBP4 receptor STRA6 and lecithin:retinol acyltransferase, which promotes selective distribution to the locus of action in the eye (Palczewski, 2010). Rational design of such retinaldehyde-sequestering compounds with the desired

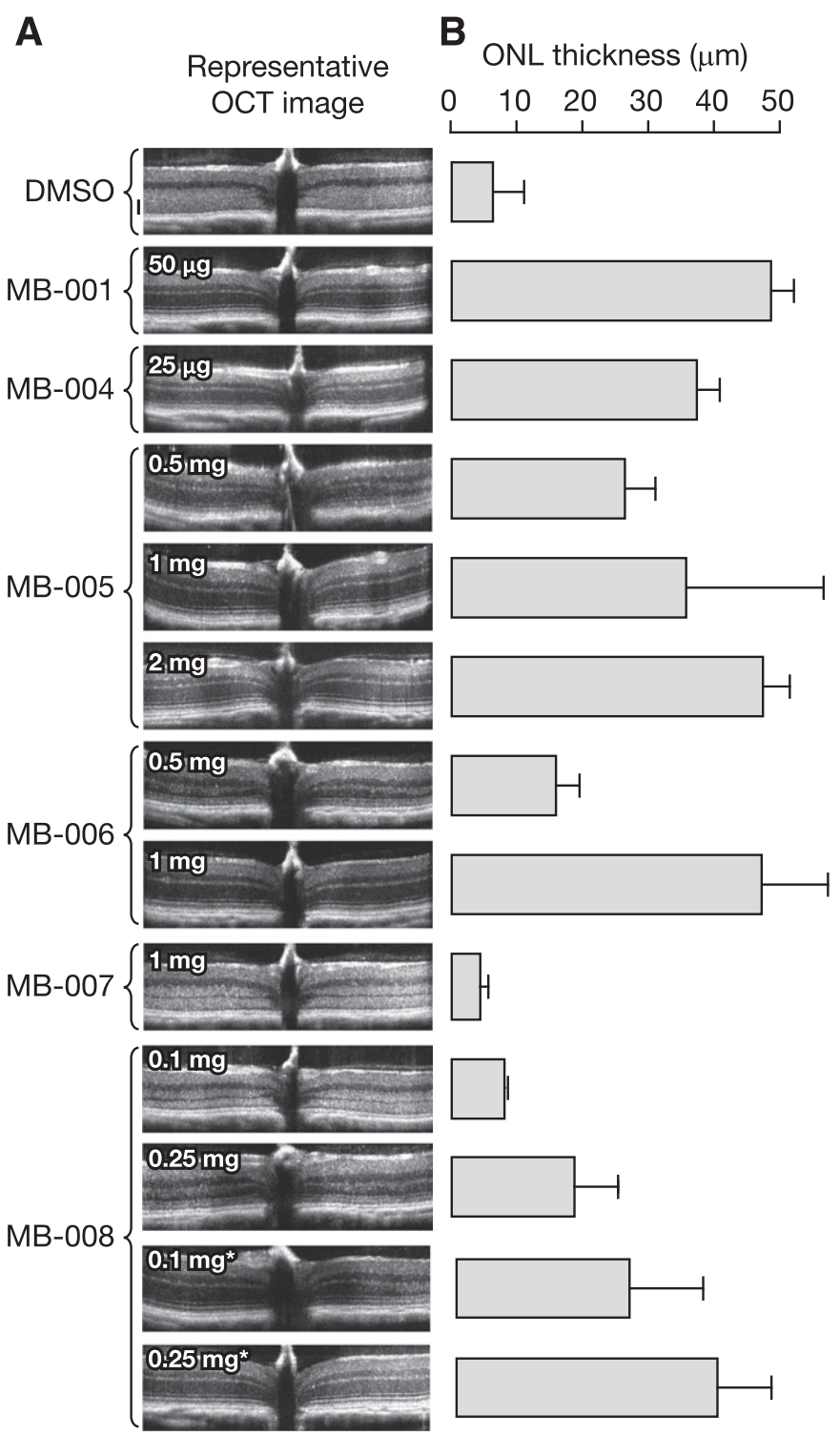

Fig. 8. Protective effects of MB compounds against light-induced retinal degeneration in $A b c a 4^{-1} R d h 8^{-1-}$ mice. Drugs were administrated at the indicated doses to 4 - to 6 -week-old $A b c a 4^{-1-} R d h 8^{--}$mice (weight $\sim 20 \mathrm{~g}$ ) by oral gavage. After a 24- or 6-hour (marked by asterisks) dark adaptation, mice were exposed to 10,000 lux light for 30 minutes and then returned to a dark environment for 3 days. (A) Representative optical coherence tomography (OCT) images of mice treated with MB compounds. A dramatic decrease in the outer nuclear layer (ONL) indicates advanced retinal degeneration. The black scale beside the dimethylsulfoxide (DMSO) image represents $50 \mu \mathrm{m}$. (B) Retinal protective effects exerted by MB compounds as quantified by average ONL thickness. Only nonscattering ONL layers were measured. For images in which the ONL layer boundary was obscured due to light scattering, the ONL length was recorded as $0 \mu \mathrm{m}$. Error bars represent standard deviations; $n=3-5$ for each group.

effect on RPE65 was facilitated by knowledge of emixustat's binding site within the RPE65 active site (Kiser et al., 2015). High-resolution structures of protein-ligand complexes have proven valuable for both examining the molecular mechanisms of drug action (Ioanoviciu et al., 2005; Illing et al., 2012; Fischer et al., 2014) and optimization of pharmacodynamic and pharmacokinetic properties (Wlodawer and Erickson, 1993; Blundell et al., 2002; Hauel et al., 2002). Such an approach eliminates a "shotgun" high-throughput medicinal chemistry search to identify chemically complex compounds 
such as retinoids, which are challenging synthetic chemistry targets. We previously used this strategy in the design of a nonprimary amine emixustat derivative, which demonstrated that the retinaldehyde-sequestering activity of emixustat is a key component of its ability to protect against retinal degeneration in mouse light-damage models (Zhang et al., 2015). In this work, we found that molecules capable of occupying a hydrophobic pocket near the active-site opening-namely, MB-004 and MB-008-exhibit superior in vitro RPE65 inhibitory activity relative to emixustat and MB-001, whereas the presence of a polar moiety in the case MB-008 did not substantially contribute to binding affinity. Binding affinity likely could be further improved through a more sophisticated analysis of the binding-pocket volume and geometry. For example, it was previously shown for hydrophobic pockets that moieties occupying $\sim 55 \%$ of the available binding space tend to be optimal concerning binding affinity (Zurcher and Diederich, 2008; Zurcher et al., 2008). The RPE65 hydrophobic pocket discussed here has a volume of approximately $154 \AA^{3}$, whereas the volume of a propyl group is $\sim 63 \AA^{3}$, suggesting that expansion of the alkyl chain length by one methylene group, which would produce a molecular volume of $\sim 79 \AA^{3}$, could further improve binding affinity.

Likewise, the binding strength of ordered waters within the hydrophilic cavity across from the aforementioned hydrophilic pocket could inform strategies for maximizing ligand interactions at this site. Weakly bound water molecules, which typically engage in three or fewer hydrogen-bonding interactions with the protein (Barillari et al., 2007), can be targeted for displacement by appropriately positioned polar ligand substituents, as release of a water molecule in this situation should be thermodynamically favorable (Michel et al., 2009). Conversely, there can be a substantial energetic cost to displacing more strongly bound waters, so in those cases, it is likely better if the ligand is designed to favorably interact with, rather than displace, the ordered water molecule. Waters contained within the RPE65 hydrophilic pocket are all engaged in three or more hydrogen-bonding interactions, two of which are formed with protein atoms and the remainder with adjacent water molecules. The binding strength of these waters likely will require detailed calculations to be accurately estimated. Suitable polar modifications could strengthen ligand binding. However, any potential gains in binding affinity could be offset by poorer membrane permeability, which would compromise bioavailability and distribution to the site of action in the RPE.

High-affinity RPE65 inhibitors could find use in the laboratory as well as the clinic. For example, such compounds could be important tools for elucidating the role of RPE65 in the visual function of species in which genetic manipulation is not currently possible-for example, those that possess conedominant retinas. Cones are thought to receive their visual chromophore from both the classic visual cycle as well as an intraretinal, Müller cell-based pathway that allows sustained regeneration under high-illumination conditions (Wang and Kefalov, 2011; Saari, 2012). The contribution of RPE65 to cone cell function in such species remains incompletely understood, although the ERG data presented here indicate that cone function recovered to a much greater extent than rod function. Selective RPE65 inhibitors could be used to eliminate classic visual cycle function in such species and allow detailed examination of the intraretinal visual cycle in isolation. For patients with Stargardt disease wherein $\mathrm{A} 2 \mathrm{E}$ is thought to be a primary contributor to macular degeneration (Sparrow and Boulton, 2005; Moiseyev et al., 2010; Charbel Issa et al., 2015; Saad and Washington, 2016), direct RPE65 inhibition by emixustat-like molecules could be therapeutically advantageous, as it was previously reported that both emixustat and its predecessor retinylamine lower $\mathrm{A} 2 \mathrm{E}$ formation in mouse models of Stargardt disease (Maeda et al., 2011; Bavik et al., 2015). These compounds also exert beneficial effects in mouse models of diabetic retinopathy thought to be related to their ability to inhibit RPE65 (Liu et al., 2015). Improving visual cycle modulator potency could lessen any off-target side effects. However, clinical trials with emixustat indicate that side effects directly related to RPE65 inhibition, including night blindness and dyschromatopsia, are most frequent and often problematic for patients (Kubota et al., 2014). Tighter binding inhibitors could have prolonged effects that are less well tolerated, whereas a molecule with a shorter duration of action, such as that of MB-008, could be advantageous for modulating the adverse effects associated with visual cycle suppression.

In a recent phase $\mathrm{IIb} / \mathrm{III}$ a clinical trial, emixustat disappointingly failed to protect against the progression of geographic atrophy in patients with dry AMD (NCT01802866). Notably, in this trial, a dose at the lower end of the originally studied range (Kubota et al., 2014) was used to minimize vision-related adverse drug reactions. The data presented here demonstrate that the ability of primary amine emixustat-like molecules to protect against light-induced retinal degeneration in mice is incompletely correlated with their ability to slow visual cycle activity. In light of these findings, the low dose of emixustat used in clinical trials could have provided insufficient retinaldehydesequestering activity, thus attenuating the retinal protective activity of emixustat, despite achieving substantial visual cycle suppression. Among the isopropyl emixustat derivatives with attenuated RPE65 inhibitory activity examined in this study, MB-005 meets many initial criteria for further development as a compound for treatment of retinal pathologies, such as AMD and Stargardt disease. This compound could be given at doses that protect mice against retinal phototoxicity with much fewer potent effects on visual cycle function as compared with emixustat. The origin of the residual effects on in vivo visual cycle function remains to be elucidated but these could arise from inhibition of visual cycle enzymes other than RPE65, including retinol dehydrogenases and lecithin:retinol acyltransferase (Kiser et al., 2014; Kiser and Palczewski, 2016). This work highlights the importance of thorough counter-screening of lead candidates at different levels, including in vivo assays to ensure productive drug development.

\section{Acknowledgments}

The authors thank Leslie T. Webster Jr. for valuable comments on the manuscript.

\section{Authorship Contributions}

Participated in research design: Kiser, Zhang, Badiee, Peachey, Tochtrop, Palczewski.

Conducted experiments: Kiser, Zhang, Badiee, Kinoshita, Peachey, Tochtrop.

Wrote or contributed to the writing of the manuscript: Kiser, Zhang, Badiee, Kinoshita, Peachey, Tochtrop, Palczewski. 


\section{References}

Barillari C, Taylor J, Viner R, and Essex JW (2007) Classification of water molecules in protein binding sites. $J$ Am Chem Soc 129:2577-2587.

Bavik C, Henry SH, Zhang Y, Mitts K, McGinn T, Budzynski E, Pashko A, Lieu KL, Zhong S, Blumberg B, et al. (2015) Visual Cycle Modulation as an Approach toward Preservation of Retinal Integrity. PLoS One 10:e 0124940.

Ben-Shabat S, Parish CA, Vollmer HR, Itagaki Y, Fishkin N, Nakanishi K, and Sparrow JR (2002) Biosynthetic studies of A2E, a major fluorophore of retinal pigment epithelial lipofuscin. J Biol Chem 277:7183-7190.

Blundell TL, Jhoti H, and Abell C (2002) High-throughput crystallography for lead discovery in drug design. Nat Rev Drug Discov 1:45-54

Brown HC, Choi YM, and Narasimhan S (1982) Selective reductions. 29. A simple technique to achieve an enhanced rate of reduction of representative organic compounds by borane-dimethyl sulfide. J Org Chem 47:3153-3163.

Calvert PD, Krasnoperova NV, Lyubarsky AL, Isayama T, Nicoló M, Kosaras B, Wong G, Gannon KS, Margolskee RF, Sidman RL, et al. (2000) Phototransduction in transgenic mice after targeted deletion of the rod transducin alpha -subunit. Proc Natl Acad Sci USA 97:13913-13918.

Caspi DD, Ebner DC, Bagdanoff JT, and Stoltz BM (2004) The resolution of important pharmaceutical building blocks by palladium-catalyzed aerobic oxidation of secondary alcohols. Adv Synth Catal 346:185-189.

Charbel Issa P, Barnard AR, Herrmann P, Washington I, and MacLaren RE (2015) Rescue of the Stargardt phenotype in Abca4 knockout mice through inhibition of vitamin A dimerization. Proc Natl Acad Sci USA 112:8415-8420.

Chen VB, Arendall, 3rdWB, Headd JJ, Keedy DA, Immormino RM, Kapral GJ, Murray LW, Richardson JS, and Richardson DC (2010) MolProbity: all-atom structure validation for macromolecular crystallography. Acta Crystallogr D Biol Crystallogr 66:12-21.

Chen VB, Wedell JR, Wenger RK, Ulrich EL, and Markley JL (2015) MolProbity for the masses-of data. J Biomol NMR 63:77-83.

Chen Y, Okano K, Maeda T, Chauhan V, Golczak M, Maeda A, and Palczewski K (2012) Mechanism of all-trans-retinal toxicity with implications for stargardt disease and age-related macular degeneration. J Biol Chem 287:5059-5069.

Crombie BS, Smith C, Varnavas CZ, and Wallace TW (2001) A conjugate additionradical cyclisation approach to sesquiterpene-phenol natural products. $J$ Chem Soc, Perkin Trans 1 206-215.

Debreczeni JE and Emsley P (2012) Handling ligands with Coot. Acta Crystallogr D Biol Crystallogr 68:425-430.

Emsley P, Lohkamp B, Scott WG, and Cowtan K (2010) Features and development of Coot. Acta Crystallogr D Biol Crystallogr 66:486-501.

Fischer ES, Böhm K, Lydeard JR, Yang H, Stadler MB, Cavadini S, Nagel J, Serluca F, Acker V, Lingaraju GM, et al. (2014) Structure of the DDB1-CRBN E3 ubiquitin ligase in complex with thalidomide. Nature 512:49-53.

Gallou F, Haenggi R, Hirt H, Marterer W, Schaefer F, and Seeger-Weibel M (2008) A practical non-cryogenic process for the selective functionalization of bromoaryls. Tetrahedron Lett 49:5024-5027.

Gohain M, du Plessis M, van Tonder JH, and Bezuidenhoudt BCB (2014) Preparation of phenolic compounds through catalyst-free ipso-hydroxylation of arylboronic acids. Tetrahedron Lett 55:2082-2084.

Golczak M, Kuksa V, Maeda T, Moise AR, and Palczewski K (2005) Positively charged retinoids are potent and selective inhibitors of the trans-cis isomerization in the retinoid (visual) cycle. Proc Natl Acad Sci USA 102:8162-8167.

Grimm C, Wenzel A, Stanescu D, Samardzija M, Hotop S, Groszer M, Naash M, Gassmann M, and Remé C (2004) Constitutive overexpression of human erythropoietin protects the mouse retina against induced but not inherited retinal degeneration. J Neurosci 24:5651-5658.

Hauel NH, Nar H, Priepke H, Ries U, Stassen JM, and Wienen W (2002) Structure-based design of novel potent nonpeptide thrombin inhibitors. J Med Chem 45:1757-1766.

Hofmann KP, Scheerer P, Hildebrand PW, Choe HW, Park JH, Heck M, and Ernst OP (2009) A G protein-coupled receptor at work: the rhodopsin model. Trends Biochem Sci 34:540-552.

Hubbard R and Wald G (1952) Cis-trans isomers of vitamin A and retinene in vision. Science 115: Unknown.

Illing PT, Vivian JP, Dudek NL, Kostenko L, Chen Z, Bharadwaj M, Miles JJ, KjerNielsen L, Gras S, Williamson NA, et al. (2012) Immune self-reactivity triggered by drug-modified HLA-peptide repertoire. Nature 486:554-558.

Ioanoviciu A, Antony S, Pommier Y, Staker BL, Stewart L, and Cushman M (2005) Synthesis and mechanism of action studies of a series of norindenoisoquinoline topoisomerase I poisons reveal an inhibitor with a flipped orientation in the ternary DNA-enzyme-inhibitor complex as determined by X-ray crystallographic analysis. J Med Chem 48:4803-4814.

Kabsch W (2010) Xds. Acta Crystallogr D Biol Crystallogr 66:125-132.

Katz ML and Redmond TM (2001) Effect of Rpe65 knockout on accumulation of lipofuscin fluorophores in the retinal pigment epithelium. Invest Ophthalmol Vis Sci 42:3023-3030.

Kimura T, Yamamoto N, Suzuki Y, Kawano K, Norimine Y, Ito K, Nagato S, Iimura $\mathrm{Y}$, and Yonaga M (2002) Practical synthesis of chiral emopamil left hand as a bioactive motif. J Org Chem 67:6228-6231.

Kiser PD, Golczak M, Lodowski DT, Chance MR, and Palczewski K (2009) Crystal structure of native RPE65, the retinoid isomerase of the visual cycle. Proc Nat Acad Sci USA 106:17325-17330.

Kiser PD, Golczak M, and Palczewski K (2014) Chemistry of the retinoid (visual) cycle. Chem Rev 114:194-232.

Kiser PD and Palczewski K (2016) Retinoids and Retinal Diseases. Annu Rev Vis Sci 2:197-234.

Kiser PD, Zhang J, Badiee M, Li Q, Shi W, Sui X, Golczak M, Tochtrop GP, and Palczewski K (2015) Catalytic mechanism of a retinoid isomerase essential for vertebrate vision. Nat Chem Biol 11:409-415.

Kubota R, Al-Fayoumi S, Mallikaarjun S, Patil S, Bavik C, and Chandler JW (2014) Phase 1, dose-ranging study of emixustat hydrochloride (ACU-4429), a novel visual cycle modulator, in healthy volunteers. Retina 34:603-609.
Kubota R, Boman NL, David R, Mallikaarjun S, Patil S, and Birch D (2012) Safety and effect on rod function of ACU-4429, a novel small-molecule visual cycle modulator. Retina 32:183-188.

Liu H, Tang J, Du Y, Lee CA, Golczak M, Muthusamy A, Antonetti DA, Veenstra AA, Amengual J, von Lintig J, et al. (2015) Retinylamine Benefits Early Diabetic Retinopathy in Mice. J Biol Chem 290:21568-21579.

Maeda A, Golczak M, Chen Y, Okano K, Kohno H, Shiose S, Ishikawa K, Harte W, Palczewska G, Maeda T, et al. (2011) Primary amines protect against retinal degeneration in mouse models of retinopathies. Nat Chem Biol 8:170-178.

Maeda A, Maeda T, Golczak M, Chou S, Desai A, Hoppel CL, Matsuyama S, and Palczewski K (2009a) Involvement of all-trans-retinal in acute light-induced retinopathy of mice. $J$ Biol Chem 284:15173-15183.

Maeda A, Maeda T, Golczak M, and Palczewski K (2008) Retinopathy in mice induced by disrupted all-trans-retinal clearance. J Biol Chem 283:26684-26693.

Maeda T, Cideciyan AV, Maeda A, Golczak M, Aleman TS, Jacobson SG, and Palczewski K (2009b) Loss of cone photoreceptors caused by chromophore depletion is partially prevented by the artificial chromophore pro-drug, 9-cis-retinyl acetate. Hum Mol Genet 18:2277-2287.

Maeda T, Golczak M, and Maeda A (2012) Retinal photodamage mediated by alltrans-retinal. Photochem Photobiol 88:1309-1319.

Maiti P, Kong J, Kim SR, Sparrow JR, Allikmets R, and Rando RR (2006) Small molecule RPE65 antagonists limit the visual cycle and prevent lipofuscin formation. Biochemistry 45:852-860.

Michel J, Tirado-Rives J, and Jorgensen WL (2009) Energetics of displacing water molecules from protein binding sites: consequences for ligand optimization. $J \mathrm{Am}$ Chem Soc 131:15403-15411.

Moiseyev G, Nikolaeva O, Chen Y, Farjo K, Takahashi Y, and Ma JX (2010) Inhibition of the visual cycle by A2E through direct interaction with RPE65 and implications in Stargardt disease. Proc Natl Acad Sci USA 107:17551-17556.

Murshudov GN, Skubák P, Lebedev AA, Pannu NS, Steiner RA, Nicholls RA, Winn MD, Long F, and Vagin AA (2011) REFMAC5 for the refinement of macromolecular crystal structures. Acta Crystallogr D Biol Crystallogr 67:355-367.

Niu J, Guo P, Kang J, Li Z, Xu J, and Hu S (2009) Copper(I)-catalyzed aryl bromides to form intermolecular and intramolecular carbon-oxygen bonds. J Org Chem 74:5075-5078.

Palczewski K (2006) G protein-coupled receptor rhodopsin. Annu Rev Biochem 75:743-767.

Palczewski K (2010) Retinoids for treatment of retinal diseases. Trends Pharmacol Sci 31:284-295.

Read RJ, Adams PD, Arendall, 3rdWB, Brunger AT, Emsley P, Joosten RP, Kleywegt GJ, Krissinel EB, Lütteke T, Otwinowski Z, et al. (2011) A new generation of crystallographic validation tools for the protein data bank. Structure 19:1395-1412.

Redmond TM, Poliakov E, Yu S, Tsai JY, Lu Z, and Gentleman S (2005) Mutation of key residues of RPE65 abolishes its enzymatic role as isomerohydrolase in the visual cycle. Proc Natl Acad Sci USA 102:13658-13663.

Redmond TM, Yu S, Lee E, Bok D, Hamasaki D, Chen N, Goletz P, Ma JX, Crouch $\mathrm{RK}$, and Pfeifer K (1998) Rpe65 is necessary for production of 11-cis-vitamin A in the retinal visual cycle. Nat Genet 20:344-351.

Rohrer B, Lohr HR, Humphries P, Redmond TM, Seeliger MW, and Crouch RK (2005) Cone opsin mislocalization in Rpe65-/- mice: a defect that can be corrected by 11-cis retinal. Invest Ophthalmol Vis Sci 46:3876-3882.

Saad L and Washington I (2016) Can vitamin A be improved to prevent blindness due to age-related macular degeneration, Stargardt disease and other retinal dystrophies? Adv Exp Med Biol 854:355-361.

Saari JC (2012) Vitamin A metabolism in rod and cone visual cycles. Annu Rev Nutr 32:125-145.

Somai Magar KB and Lee YR (2014) Ruthenium(II)-catalyzed protocol for preparation of diverse $\alpha, \beta$ - and $\beta, \beta$-dihaloenones from diazodicarbonyls. Adv Synth Catal 356:3422-3432. Sparrow JR and Boulton M (2005) RPE lipofuscin and its role in retinal pathobiology. Exp Eye Res 80:595-606.

Sparrow JR, Fishkin N, Zhou J, Cai B, Jang YP, Krane S, Itagaki Y, and Nakanishi K (2003) A2E, a byproduct of the visual cycle. Vision Res 43:2983-2990.

Stecher H, Gelb MH, Saari JC, and Palczewski K (1999) Preferential release of 11-cisretinol from retinal pigment epithelial cells in the presence of cellular retinaldehyde-binding protein. J Biol Chem 274:8577-8585.

Wald G (1968) The molecular basis of visual excitation. Nature 219:800-807.

Wang JS and Kefalov VJ (2011) The cone-specific visual cycle. Prog Retin Eye Res $\mathbf{3 0}$ 115-128.

White WN and Norcross BE (1961) The ortho-claisen rearrangement. III. The rearrangement of $\beta$-alkylallyl aryl ethers1,2. J Am Chem Soc 83:3265-3269.

Wlodawer A and Erickson JW (1993) Structure-based inhibitors of HIV-1 protease. Annu Rev Biochem 62:543-585.

Wu J, Peachey NS, and Marmorstein AD (2004) Light-evoked responses of the mouse retinal pigment epithelium. J Neurophysiol 91:1134-1142.

Zav'yalov SI, Ezhova GI, Sltkareva IV, Dorofeeva OV, Zavozin AG, and Rumyantseva $\mathrm{EE}$ (1989) $\mathrm{Br} 2-\mathrm{CO}(\mathrm{NH} 2) 2-\mathrm{AcOH}$ system for the highly selective $\alpha$-monobromination of ketones. B ACAD SCI USSR CH 38:2204-2204.

Zhang J, Kiser PD, Badiee M, Palczewska G, Dong Z, Golczak M, Tochtrop GP, and Palczewski K (2015) Molecular pharmacodynamics of emixustat in protection against retinal degeneration. $J$ Clin Invest 125:2781-2794.

Zürcher M and Diederich F (2008) Structure-based drug design: exploring the proper filling of apolar pockets at enzyme active sites. J Org Chem 73:4345-4361.

Zürcher M, Gottschalk T, Meyer S, Bur D, and Diederich F (2008) Exploring the flap pocket of the antimalarial target plasmepsin II: the " $55 \%$ rule" applied to enzymes. ChemMedChem 3:237-240.

Address correspondence to: Philip Kiser, Department of Pharmacology, School of Medicine, Case Western Reserve University, 10900 Euclid Avenue, Cleveland, OH 44106. E-mail: pdk7@case.edu or Krzysztof Palczewski, Department of Pharmacology, School of Medicine, Case Western Reserve University, 10900 Euclid Avenue, Cleveland, OH 44106. E-mail: kxp65@case.edu 